\title{
Preventing and treating kidney disease in patients with type 2 diabetes
}

\section{Pierre Delanaye \& André J. Scheen}

To cite this article: Pierre Delanaye \& André J. Scheen (2019) Preventing and treating kidney disease in patients with type 2 diabetes, Expert Opinion on Pharmacotherapy, 20:3, 277-294, DOI: 10.1080/14656566.2018.1551362

To link to this article: https://doi.org/10.1080/14656566.2018.1551362

Accepted author version posted online: 21

Nov 2018.

Published online: 03 Dec 2018.

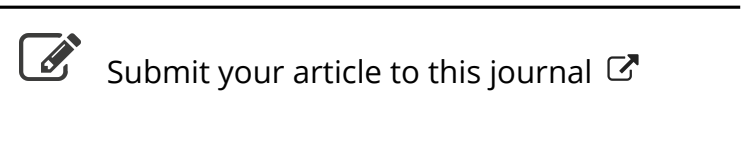

LII Article views: 146

View Crossmark data ¿ 


\title{
Preventing and treating kidney disease in patients with type 2 diabetes
}

\author{
Pierre Delanaye ${ }^{\mathrm{a}}$ and André J. Scheen ${ }^{\mathrm{b}, \mathrm{c}}$
}

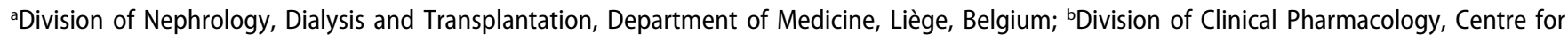
Interdisciplinary Research on Medicines (CIRM), University of Liège, Liège, Belgium; 'Department of Medicine, Division of Diabetes, Nutrition and Metabolic Disorders, Liège, Belgium

\begin{abstract}
Introduction: Chronic kidney disease (CKD) represents a huge burden in patients with type 2 diabetes (T2DM). This review therefore has the aim of assessing the add-on value of new glucose-lowering agents compared or combined with inhibitors of the renin angiotensin aldosterone system (RAAS) on renal outcomes in T2DM patients.

Areas covered: This article first summarizes the results reported with RAAS inhibitors, mainstay of nephroprotection in T2DM with albuminuria. Second, it describes the positive results with glucagon-like peptide-1 receptor agonists (GLP-1RAs) and, even more impressive, sodium-glucose cotransporter type 2 inhibitors (SGLT2is). Third, besides the potential of combined therapies, it briefly considers some new approaches currently in development.

Expert opinion: RAAS inhibitors exert renoprotective effects beyond their blood pressure lowering effects while SGLT2is, and possibly GLP-1RAs, exert nephroprotection independently of their glucoselowering activity. These effects were demonstrated not only on surrogate endpoints such as albuminuria and estimated glomerular filtration rate decline, but also on hard endpoints, including progression to end-stage renal disease requiring replacement therapy. The underlying mechanisms are different and potentially complementary on glomerular hemodynamics, arguing for combined therapies. Nevertheless, there is still room for new emerging drugs to tackle CKD in T2DM.
\end{abstract}

ARTICLE HISTORY

Received 16 August 2018

Accepted 19 November

2018

\section{KEYWORDS}

Chronic kidney disease; GLP1 receptor agonist; RAS inhibitors; SGLT2 inhibitor; Type 2 diabetes

\section{Introduction}

Chronic kidney disease (CKD) is a classical complication of diabetes mellitus, which may lead to end-stage renal disease (ESRD) [1]. Its pathophysiology is more complex in type 2 diabetes (T2DM) than in type 1, especially because of the intervention of several other risk factors beyond chronic hyperglycemia: arterial hypertension, abdominal obesity, dyslipidemia, hyperuricemia, which all are associated with insulin resistance, low-grade inflammation and oxidative stress [2,3]. Globally, the burden of diabetes and hypertension considered as the two leading drivers of CKD has increased dramatically worldwide over the past several decades. The number of patients with known diabetes quadrupled between 1980 and 2014 while the number of adults with elevated blood pressure almost doubled between 1975 and 2014. Globally, CKD due to diabetes and CKD due to hypertension contributed to about half and near a quarter of the overall increase in CKD disability-adjusted-life-years, respectively [4]. Hypertension and diabetes are coexisting in the vast majority of patients with T2DM. Making a clear distinction between these two entities (patient primarily hypertensive or diabetic) might be difficult but pragmatically little relevant from a therapeutic point of view. This is illustrated by the fact that a kidney biopsy to prove diabetic nephropathy was not required in all large trials that will be discussed hereafter. The annual incidence of microalbuminuria and albuminuria in patients with T2DM averaged $\sim 8 \%$, with an incidence of developing estimated glomerular filtration rate (eGFR) using the Modification of Diet on Renal Disease (MDRD) equation - $<60 \mathrm{ml} / \mathrm{min} / 1.73 \mathrm{~m}^{2}$ estimated to be $\sim 2-4 \%$ per year and an annual incidence of ESRD ranging from $0.04 \%$ to $1.8 \%$ [5]. Over the last two decades, the prevalence of CKD is increasing in patients with T2DM, a paradoxical effect of the better management of cardiovascular risk factors that increases life expectancy in this high-risk population [6]. Thus, both the prevention and early appropriate management of CKD in patients with T2DM currently represents a major challenge $[7,8]$.

Tight glucose control is the first step to prevent microangiopathy, whose early renal biological marker is the development and progression of albuminuria [9]. Evidence also suggests that it can ameliorate eGFR loss and possibly progression to ESRD, yet these benefits appear to be most pronounced when applied to T2DM patients with the early stages of CKD [10]. However, tight glucose control is not easy to reach and maintain in many T2DM patients, requires longterm follow-up to prove its efficacy on hard clinical endpoints and may be insufficient to improve renal outcomes, especially when CKD is already present [11]. Furthermore, CKD may alter the pharmacokinetic parameters of several glucose-lowering agents, an effect that renders their use more difficult or even contraindicated in clinical practice $[12,13]$.

Lowering arterial blood pressure also reduces renal events in patients with T2DM [14]. This effect may be additive to the 
Article Highlights

- Glucose and blood pressure control are essential to prevent CKD in T2DM, an increasing prevalent complication of the disease.

- RAAS blockade, either with ACE inhibitor or ARA II, is the cornerstone of nephroprotection in T2DM, but dual blockade should be avoided for safety reasons.

- Metformin may now be used in patients with CKD (if eGFR > $30 \mathrm{ml} /$ min per $1.73 \mathrm{~m}^{2}$ ) and might be associated with a reduced risk of progression of renal impairment.

- New glucose-lowering agents, that is, GLP-1RAs and SGLT2 inhibitors, exert renoprotective effects beyond improvement of glucose control, in combination with RAAS blockers, in T2DM patients at high cardiovascular risk.

- Because SGLT2is reduce hard renal outcomes and not only surrogate endpoints (albuminuria) as GLP-1RAs, they are considered as the best option after metformin in T2DM patients with CKD provided that eGFR is adequate.

- RAAS blockers and SGLT2 inhibitors exert different effects that can protect kidney function, yet the complementary effects on intrarenal hemodynamics appear predominant.

- To reduce the residual risk, new medications are in development targeting low-grade inflammation, oxidative stress, and renal fibrosis, as add-on therapies to existing drugs.

This box summarizes key points contained in the article.

positive effect of intensive glucose control as shown in the ADVANCE trial that tested both strategies [15]. Inhibition of the renin-angiotensin-aldosterone system (RAAS) has proven its ability to prevent and slow down the progression of CKD in diabetic patients, an effect being partially independent of the effect on arterial blood pressure [16]. The benefit is particularly prominent in patients with micro- or macro-albuminuria. According to a network meta-analysis comparing efficacy and safety of blood-pressure lowering agents in adults with diabetes and CKD, RAAS inhibitors were the most effective strategies against ESRD [17].

As add-on therapy, new glucose-lowering agents, especially sodium-glucose cotransporter type 2 inhibitors (SGLT2is) [18-21], showed beneficial renal outcomes in T2DM patients with established cardiovascular disease [22,23]. Glucagon-like peptide-1 receptor agonists (GLP-1RAs) also showed positive effects on composite renal outcomes in patients with T2DM [24,25]. These newer glucose-lowering approaches seem to be promising for the prevention and treatment of CKD in patients with T2DM, an effect occurring beyond improvement of glucose control $[13,26,27]$.

Increasing evidence showed that oxidative stress and lowgrade inflammation play a major role in diabetic complications; furthermore, renal fibrosis is an emerging entity that contribute to the early development of diabetic kidney disease. These new concepts pave the road to innovative pharmacological approaches currently in development $[28,29]$. The notion of diabetic nephropathy as a purely vascular disease is outdated and it has become clear that it is a multidimensional, multicellular condition [29].

The aim of the present narrative review is to analyze the effects of the different pharmacological therapies used to prevent or retard the progression of CKD in patients with T2DM. We will successively summarize (i) the effects of inhibitors of the RAAS, the best validated pharmacological approach regarding nephroprotection, (ii) describe the effects of new glucose lowering agents that recently showed promising results on surrogate and clinical renal outcomes, and finally (iii) introduce future therapies currently in development that target innovative mechanisms. As a main objective, the respective add-on value of SGLT2is and GLP-1RAs on renal outcomes will be discussed, both in comparison and in combination with RAAS inhibitors.

\section{Inhibitors of the renin-angiotensin-aldosterone system}

Blood pressure control is beneficial for both cardiovascular and renal outcomes in T2DM patients [30,31], as it is clearly emphasized in different guidelines in the fields of nephrology [32], diabetes [9], or hypertension [33]. More debated is whether the target levels should be different in patients with and without T2DM [33-36]. It is classically recognized that RAAS inhibition therapies have potential added value compared with other antihypertensive therapies in T2DM patients, especially in patients with (micro)albuminuria [9,32,33]. However, even if RAAS inhibitors have proven some superiority in terms of renal outcomes compared to other antihypertensive agents [17], we must keep in mind that more than one medication will be necessary to adequately control arterial blood pressure in a majority of T2DM patients with hypertension [37,38]. Of note, although beyond the scope of this review paper devoted to renal outcomes, RAAS inhibitors exert potential benefits on other clinically relevant outcomes, notably by reducing the incidence of major cardiovascular events [38-40].

RAAS inhibition can lead to renal protection in an independent way of blood pressure control via different pathways. One of the most important is certainly the effect of RAAS inhibitors on the intraglomerular pressure. Indeed, RAAS inhibitors decreased intraglomerular hypertension by limiting the vasoconstriction of postglomerular arteriole induced by angiotensin II (Figure 1). This effect will lead to a decrease in albuminuria and, at long term, to beneficial effects on kidney function [41]. This so-called 'hemodynamic' effect also explains why starting therapy with RAAS inhibitors frequently goes with an initial slight increase in serum creatinine or decrease in eGFR. This functional decrease in renal filtration is generally limited and reversible. Other mechanisms of action have been suggested to explain renal damage when the RAAS system is activated: increased oxidative stress, endothelial dysfunction, promotion of inflammation, mesangial cell proliferation, and transforming growth factor beta (TGF- $\beta$ ) profibrotic effect $[42,43]$.

Tables 1 and 2 reviewed some important randomized controlled trials (RCTs) comparing different RAAS inhibitors with placebo or other antihypertensive therapies, respectively. We will limit our analysis to studies with the largest samples (> 250 patients in each arm), focusing on patients with T2DM and, if possible, reporting 'hard' clinical renal endpoints (i.e. doubling of serum creatinine and/or ESRD, usually defined as need for dialysis or renal transplantation).

\subsection{Angiotensin converting enzyme inhibitors}

The specific role of ACE inhibitors has been first studied in type 1 diabetic patients. It is considered nowadays as the classical therapy for type 1 diabetic nephropathy, already from the early stage of 

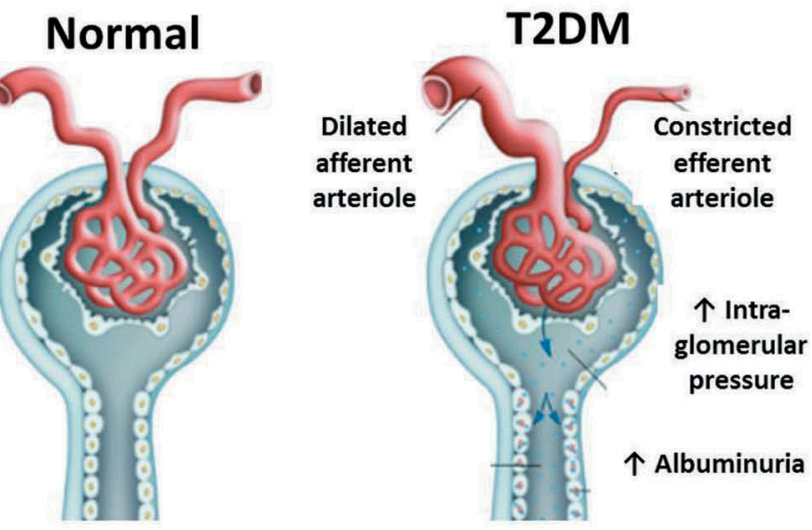

RAAS inhibition

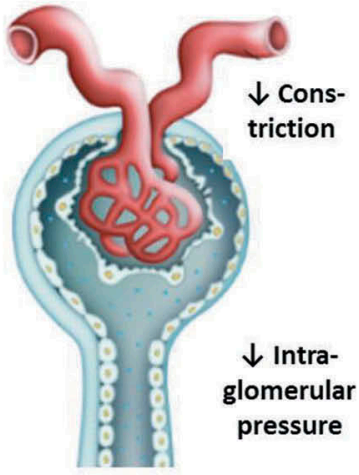

SGLT2 inhibition

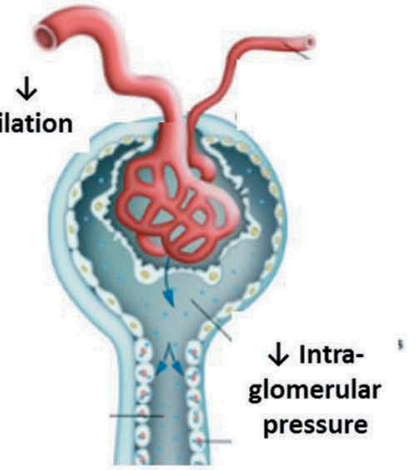

\section{RAAS inhibition + SGLT2 inhibition in T2DM patients}

$\begin{array}{cc}\begin{array}{c}\text { Relative } \\ \text { constriction of } \\ \text { afferent arteriole }\end{array} & \begin{array}{c}\text { Relative dilation } \\ \text { of efferent } \\ \text { arteriole }\end{array}\end{array}$

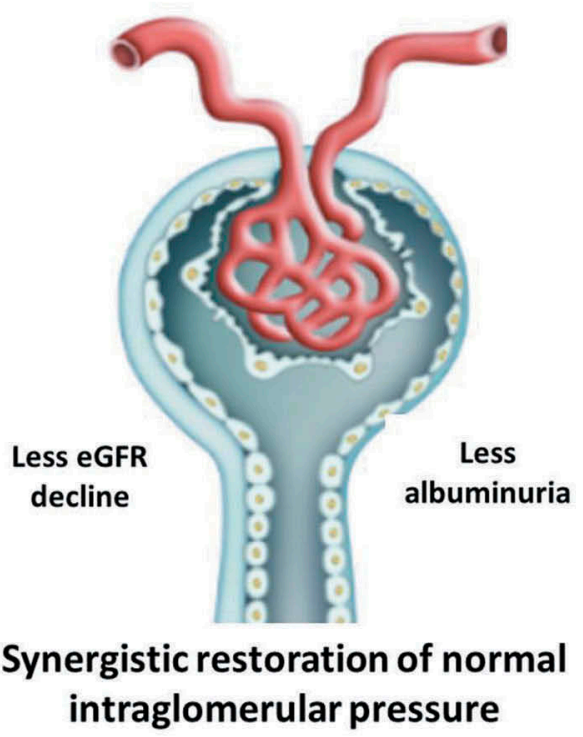

Figure 1. Complementary renoprotective effects of RAAS inhibitors and SGLT2 inhibitors by targeting efferent (through blockade of angiotensin 2 vasoconstriction) and afferent (through restoration of tubuloglomerular feedback) arterioles, respectively.

kidney damage, that is, in patients with normal GFR but presence of microalbuminuria [9]. By analogy, the indication of ACE inhibitors in T2DM patients with albuminuria is now largely adopted, even in absence of hypertension but presence of (micro-)albuminuria [9]. However, most of the studies in patients with T2DM were performed in hypertensive patients. This potential confounding factor makes the definitive proof of a positive blood pressureindependent effect of ACE inhibitors more challenging. This is particularly the case in comparative studies 'versus placebo', as the RAAS inhibitor group has frequently a better control of blood pressure. Most of the studies showed positive impacts on usual surrogate markers such as change of proteinuria (or new onset of albuminuria) and decline in GFR (or increase in serum creatinine). Specific studies with renal 'hard' end-points in T2DM patients are less numerous, and some of them are underpowered, with rather few events because a baseline low risk (normal eGFR with no or only marginal microalbuminuria) and/or with an insufficient follow-up (Table 1). For instance, in the UKPDS study, despite a long follow-up of 9 years, no significant differences could be detected between captopril and atenolol when considering the progression to microalbuminuria, macroproteinuria or ESRD (very few events); indeed, these results were obtained in patients with newly diagnosed T2DM and hypertension, but normal kidney function and low proportion of albuminuria at baseline, and reaching similar blood pressure control with both antihypertensive medications [44]. Two large prospective long-term trials, ALLHAT [45] and
ADVANCE [40], have reported data on renal endpoints, including 'hard' outcomes. In ALLHAT, an ACE inhibitor (lisinopril) was not better than a diuretic (chlorthalidone, prespecified head-to head comparison with statistical analysis) or a calcium channel blocker (amlodipine, but no head-to-head prespecified statistical analysis) on the renal composite outcomes (or the single endpoint ESRD) (Table 1). The absence of significant difference in ESRD between lisinopril and either chorthalidone or amlodipine was confirmed after an extended follow-up of about 9 years in the diabetic population of ALLHAT [46]. The impact of ALLHAT is, however, limited by the absence of any data on albuminuria and by the fact that the blood pressure control was different in the therapeutic groups $[45,46]$. ADVANCE compared an association of perindopril and indapamide versus placebo in patients with normal renal function, and only a minority had micro- or macroalbuminuria (25\% and $3.5 \%$, respectively) [40]. The composite renal outcome was significantly better in the perindopril group than in the placebo group, but the results were mainly explained by the positive effect on new onset of microalbuminuria, whereas the impact on doubling serum creatinine or ESRD was not significant in this population with a relatively low ESRD risk (Table 1). As in ALLHAT, the results could be explained, at least in part, by a better blood pressure control in the active group [14,40], but possibly also by the concomitant prescription of indapamide, a thiazide-like diuretic that showed some positive vascular and renal effects [47]. 


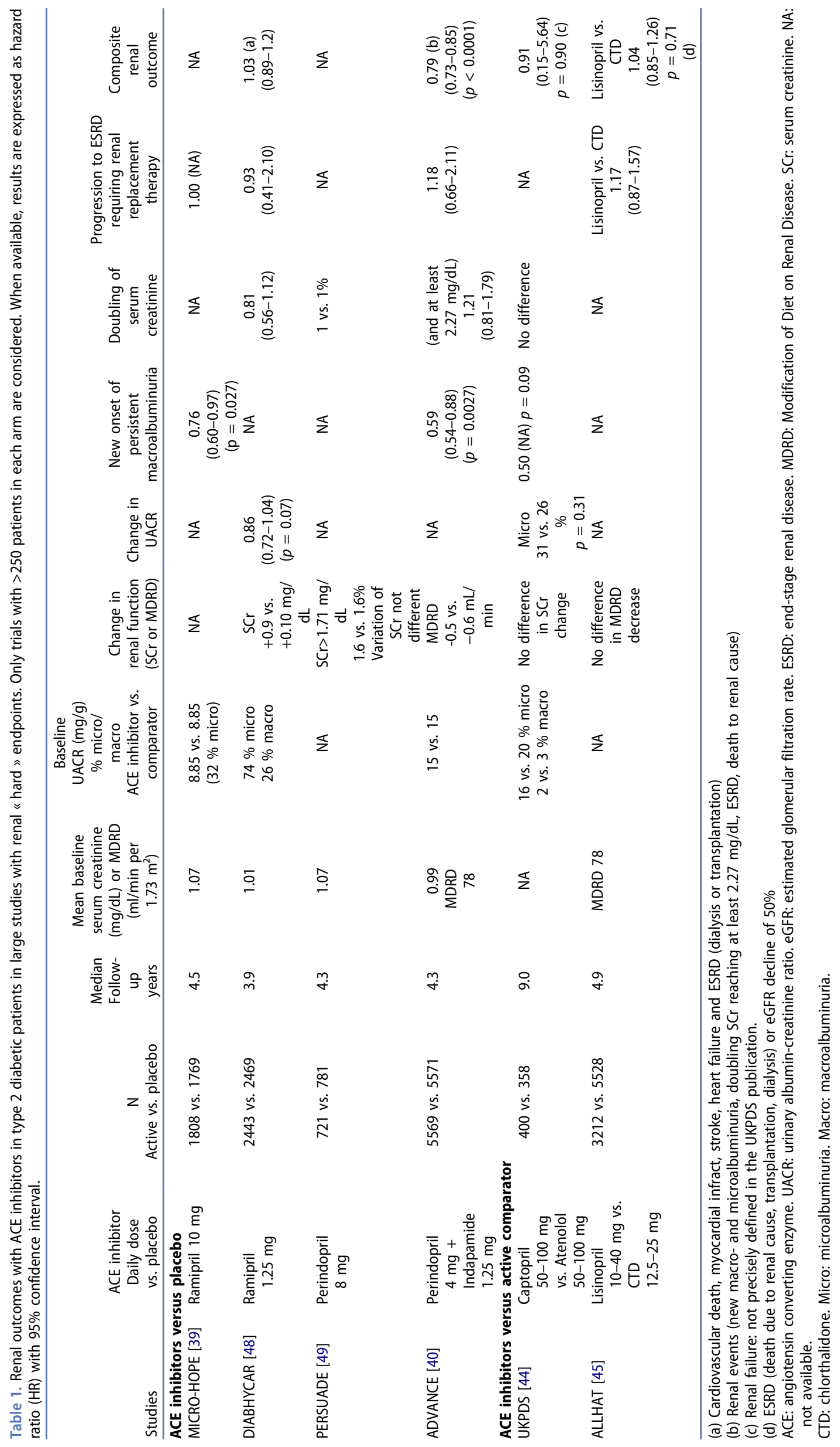


In three other studies in T2DM patients with normal serum creatinine levels (MICROHOPE, DIABHYCAR, PERSUADE), no significant differences in the changes of serum creatinine or in renal outcomes were reported when comparing an ACE inhibitor with a placebo $[39,48,49]$. However, in MICROHOPE (32\% of T2DM patients with microalbuminuria at baseline) [39] and in DIABHYCAR (74\% of T2DM patients with microalbuminuria and $26 \%$ with macroalbuminuria at baseline) [48], the rate of new onset of persistent macroalbuminuria was significantly lower and the rate of regression from macro to micro or from micro to normal albuminuria was numerically higher in the ACE inhibitor group compared with the placebo group, respectively. No data on albuminuria neither at baseline nor at the end of the trial were available in PERSUADE, an analysis that evaluated a large subgroup of T2DM patients from the cardiovascular EUROPA study [49] (Table 1).

Several meta-analyses (however, most of them mixing patients with type 1 and type 2 diabetes) support the use of ACE inhibitors in diabetic patients with renal diabetic nephropathy, especially in the presence of significant albuminuria $[34,50,51]$. The specific (independent of any blood pressure effect) added value of ACE inhibitors in the absence of albuminuria remains more debatable [52,53]. Also, data in T2DM patients with low or very low GFR values are scarce [35].

\subsection{Angiotensin II receptor antagonists}

Evidence of the role of angiotensin II receptor antagonists (ARA II) in T2DM patients is more robust than for ACE inhibitors. Two big trials focusing on renal events (with no equivalent in terms of patient characteristics and sample size for ACE inhibitors) are available with ARA II in T2DM patients: IDNT [37] and RENAAL [38] (Table 2). Both studies included a majority of hypertensive patients with already advanced CKD (elevated mean serum creatinine around $1.65 \mathrm{mg} / \mathrm{dL}$ and $1.9 \mathrm{mg} / \mathrm{dL}$ and significant (macro) albuminuria around $1.9 \mathrm{~g} /$ day and $1.2 \mathrm{~g} / \mathrm{g}$ in IDNT and RENAAL, respectively) $[37,38]$. IDNT compared irbesartan versus placebo versus amlodipine [37]. RENAAL compared losartan with placebo [38]. In both studies, the reduction effect on albuminuria was significantly better in the ARA II groups. Still more relevant, the risk of renal composite outcome was significantly lower with ARA Il compared with placebo (or even amlodipine). Also, the specific risk of doubling serum creatinine was significantly lower with ARA II in the two studies, whereas the reductions of the risk of reaching ESRD were borderline in IDNT ( $p=0.07)$ and significant in RENAAL $[37,38]$ (Table 2). This effect seems independent of blood pressure control. Other studies comparing ARA II and placebo focused on diabetic nephropathy, but in patients with a baseline lower renal risk: normal (or near to normal) renal function, well- controlled hypertension, and normoalbuminuria (or only a slight proportion of microalbuminuria) [51,53]. If some results suggest positive results in terms of albuminuria (notably on the new onset of albuminuria), results are much less evident in terms of 'hard' clinical endpoints in this population with less advanced disease and at much lower risk to progress to ESRD, as shown in the ROADMAP trial comparing olmesartan with placebo [54] (Table 2). In the Japanese study CASE-J evaluating a subgroup of T2DM patients, the ARA II candesartan was associated with a significantly lower rate of a composite renal outcome compared with the calcium channel blocker amlodipine after a follow-up of 3.3 years [55], thus confirming the results of INDT that compared irbesartan with amlodipine (Table 2). Unfortunately, no data on the albuminuria status at baseline and during the study were reported in CASE-J trial [55].

The question of the superiority of ARA II compared to ACE inhibitors has been debated for a long time, but today, most authors agree on a similar effect of the two classes of RAAS inhibitors. In a head-to-head comparative RCT in T2DM patients with albuminuria, a similar effect on albuminuria and GFR decline was observed with telmisartan and enalapril [56]. The large trial ONTARGET, although not specifically dedicated to T2DM patients (9612 diabetics among 25,620 patients included) did not show any superiority in terms of renal endpoints (GFR decline, albuminuria) but also 'hard' endpoints (such as doubling serum creatinine or reaching ESRD) of telmisartan compared with ramipril [57]. Also, pooled analyses, including network meta-analyses, do not support any clear superiority of ARA II over ACE inhibitors in T2DM patients $[34,50,52,58,59]$.

\subsection{Dual blockade of RAAS}

High-risk diabetic patients, as those included in the RENAAL or IDNT studies, had a lower albuminuria with ARA II and a better renal outcome, yet a rather high 'renal residual risk' may persist [60]. Based on first results showing an additional decrease of albuminuria with dual RAAS blockade [59], studies were carried out in high renal risk patients with therapies combining an ACE inhibitor and an ARA II (Table 3). ORIENT [61] compared renal outcomes with ACE inhibitor plus olmesartan versus ACE inhibitor plus placebo while VA-NEPHRON D [62] compared lisinopril plus losartan versus lisinopril plus placebo. Both studies included T2DM patients with macroalbuminuria and moderately decreased renal function. In both trials, a significantly better control of albuminuria was observed, but with only marginal effects on other renal outcomes (Table 3). Safety was a concern with a higher risk of dialysis and hyperkalemia in the combined therapy group [62] These data, and data from metaanalyses, confirm the absence of clear added value of ACE inhibitor- ARA II combined therapy on renal events $[17,50]$.

A similar conclusion may be drawn for aliskiren a direct renin inhibitor whose effect was investigated as add-on therapy to an ARA II or an ACE inhibitor. Preliminary encouraging results (better control of albuminuria in the aliskiren group compared with placebo) were not confirmed in the largest ALTITUDE study [63]. Even if patients had a slightly better control of albuminuria, the effect was not significant on the renal composite endpoint (and on secondary renal endpoints such as doubling serum creatinine and ESRD) (Table 3). Moreover, again safety was a concern, notably with a significantly higher risk of hyperkalemia [63].

Another way to reinforce the RAAS blockade is to add an aldosterone receptor blocker to either an ACE inhibitor or an ARA II. Several studies have been performed with the classical non-selective aldosterone antagonist spironolactone with some positive results, as summarized in several metaanalyses [64,65]. However, up to now, spironolactone has 


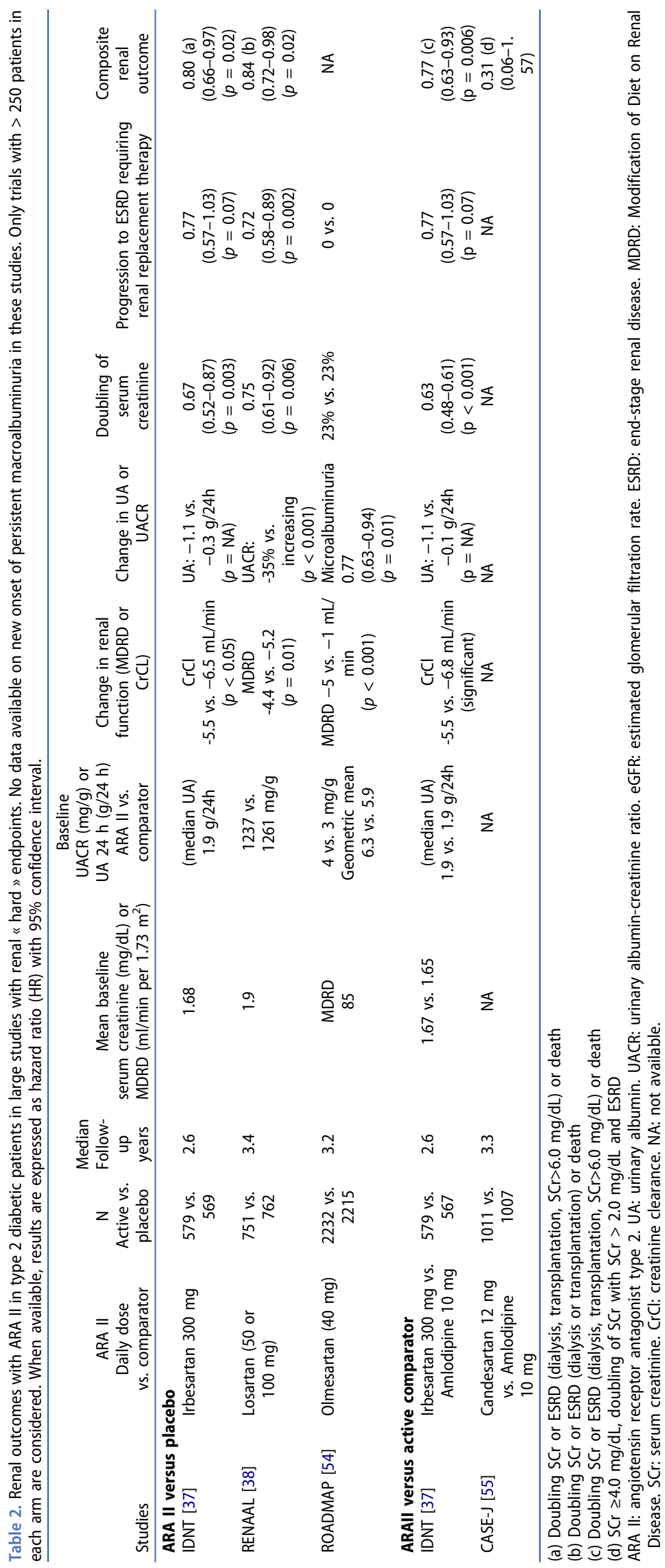




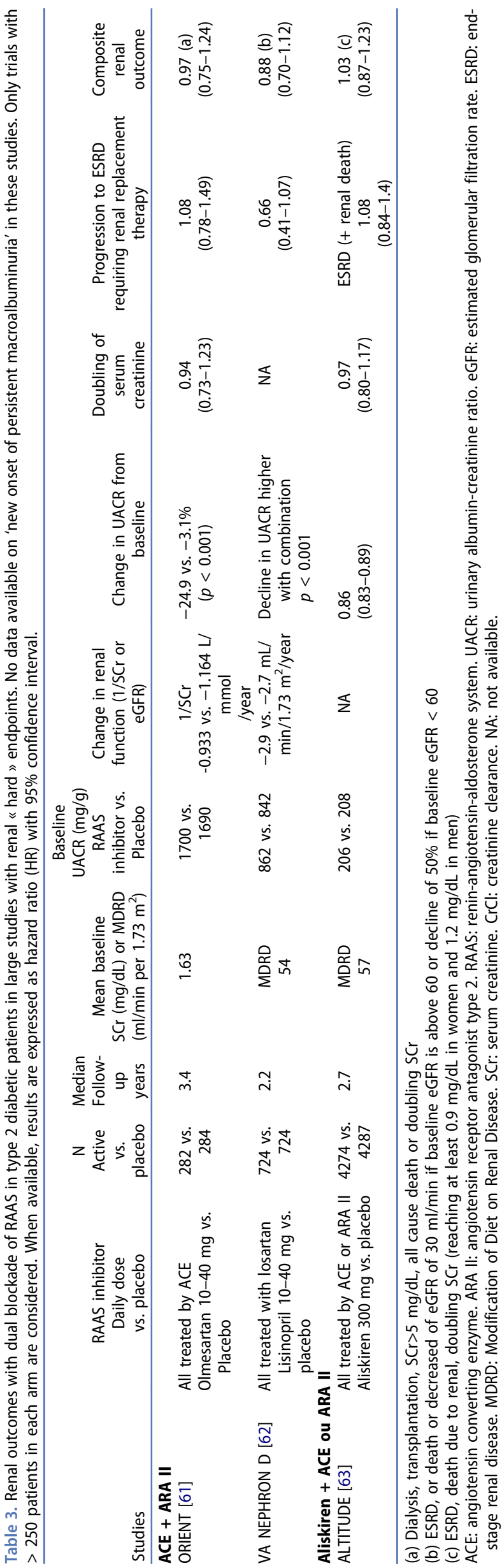

never crossed the border of limited sample size studies with surrogate markers as endpoints (principally effect on albuminuria). Reduction in albuminuria were also reported with two other mineralocorticoid receptor antagonists eplerenone [66] and finerenone [67]. Some concerns were reported with spironolactone in terms of risk of hyperkalemia, an adverse event that may occur more frequently in real life than in RCTs [68]. Thus, this association to protect renal function is not recommended in clinical practice yet. Larger prospective trials are underway, mainly with finerenone, a more selective nonsteroidal mineralocorticoid receptor antagonist that might induce less hyperkalemia $[67,69]$.

\subsection{Still for debate}

Beyond their potential benefit on blood pressure control and cardiovascular endpoints, ARA II are the drugs with the better evidence of renal protection in T2DM patients with albuminuria $[37,38,70]$. Because the evidence of a strong renal protection in type 1 diabetic patients with ACE inhibition [9], and the absence of clear superiority of ARA II compared to ACE inhibitors $[9,56]$, both pharmacological classes are now equally considered as 'standard of care' of diabetic nephropathy with albuminuria, and this is clearly stated in several guidelines $[9,32,33,35]$. Having said that, some points and 'gray zones' need to be briefly discussed. As already mentioned, the blood pressure control is of main importance in T2DM patients and this goal requires a combination of several different antihypertensive agents in a majority of patients with CKD and albuminuria [9]. The additional benefit of RAAS inhibitors, beyond blood pressure control, is mainly proven in T2DM patients with micro- and, still more, macro-albuminuria and is more debatable in absence of (micro)albuminuria [9,32]. RAAS inhibitors reduce proteinuria, a risk marker for renal disease progression and their antiproteinuric effect correlates with their additional renal benefits [71]. Data emerged from clinical trials demonstrating that use of 'supratherapeutic doses' of RAAS inhibitors (doses greater than those approved for lowering blood pressure), compared with standard doses, has favorable efficacy in reducing proteinuria in T2DM patients with CKD. Supratherapeutic dosing may be a valuable approach for optimizing RAAS blockade and providing renoprotection [72].

The benefit/risk ratio of this RAAS blocker therapy has been questioned in patients older than 70 years (such aged patients being excluded from large RCTs discussed above and presented in Table 1-3), especially in people with relatively preserved GFR and low albuminuria [73]. Renal safety (risk of hyperkalemia and acute renal failure) remains a concern, especially beyond the reassuring context of RCTs [68]. Indeed, in real life, RAAS blockade is considered as the main of cause of hospitalizations for hyperkalemia, and it is estimated that around $10 \%$ of patients will develop hyperkalemia within the first year after starting RAAS blockade therapy [74]. CKD patients have a higher risk of hyperkalemia, and this risk is dependent on the renal function (the lower the eGFR, the higher the risk), being particularly high in patients with GFR below $30 \mathrm{~mL} /$ $\min / 1.73 \mathrm{~m}^{2}$. Diabetes in itself is also a risk of hyperkalemia, as diabetic patients have hyporeninemic hypoaldosteronism and insulin defect may impair the shift of potassium from plasma 
into cells. Other important risk factors for hyperkalemia are advanced age, heart failure, volume depletion, and concomitantly use of other drugs interfering with renal excretion of potassium (for example, trimethoprim or nonsteroidal anti-inflammatory drugs). The risk will be particularly relevant in patients combining risks, typically the elderly treated by RAAS inhibitor and antiinflammatory agents and suffering from acute diarrhea. The risk of hyperkalemia is also dependent on the dose of ACE inhibitor or ARA II $[74,75]$. The risk profile of acute renal failure mirrors the risk profile of hyperkalemia and both conditions are frequently concomitant. In patients with abnormally high potassium level, it seems unreasonable to start therapies blocking RAAS. In RENAAL, including T2DM patients with $C K D$, the mean potassium concentration at baseline was $4.6 \mathrm{mmol} / \mathrm{L}$ and losartan was associated with a mean increase of up to $0.3 \mathrm{mmol} / \mathrm{l}$ [76] However, more than considering a potassium concentration threshold to start or not RAAS blocking therapy, it seems very important to monitor potassium in high risk patients and applying preventive actions [74,75], including the use of potassium binders [77].

Also for safety reasons, the dual RAAS blockade, especially the combination of ARA II and ACE inhibitors, is not recommended. Their use might be discussed in very specific patients with uncontrolled massive albuminuria, under the supervision of a nephrologist [42]. Lastly, because the hemodynamic effect of RAAS inhibitors, therapy interruption can significantly increase GFR level, a mirror image of the decline in eGFR generally observed at the initiation of a RAAS inhibitor. Thus, stopping ACE inhibitors or ARA II may be discussed in very specific patients with advanced renal failure (GFR below $20-30 \mathrm{~mL} / \mathrm{min} / 1.73 \mathrm{~m}^{2}$ ) with the aim of delaying dialysis [78].

\section{Glucose-lowering medications}

Most antihyperglycemic medications can be used safely in patients with mild to moderate CKD. However, several glucoselowering agents are either not advised or require dose adjustments in more advanced CKD [12,79]. Of note, regulations guiding the use of metformin, the first-line treatment for the pharmacological management of T2DM, in patients with stable, moderate renal dysfunction have become more lenient in recent years and metformin may be used at half dose in patients with eGFR between 30 and $45 \mathrm{ml} / \mathrm{min} / 1.73 \mathrm{~m}^{2}$. In the American recommendations, no precise dosages are recommended in cases of renal impairment, but metformin should not be initiated if eGFR is below $45 \mathrm{ml} / \mathrm{min} / 1.73 \mathrm{~m}^{2}$. In Europe, the maximum daily metformin recommended dose is $2000 \mathrm{mg} /$ day in CKD stage 3a (eGFR $=45-59 \mathrm{ml} / \mathrm{min} / 1.73 \mathrm{~m}^{2}$ ) and $1000 \mathrm{mg} /$ day in CKD stage $3 \mathrm{~b}\left(\mathrm{GFR}=30-44 \mathrm{ml} / \mathrm{min} / 1.73 \mathrm{~m}^{2}\right)$. There is agreement upon the fact that metformin must be stopped if eGFR consistently falls below $30 \mathrm{ml} / \mathrm{min} / 1.73 \mathrm{~m}^{2}$ [80]. Also the concurrent withdrawal (albeit temporarily) of metformin is recommended when conditions leading to intermittent hypovolemic state are present, circumstances that also should lead to the transient interruption of RAAS inhibitors [81].

Intensive glucose control with classical glucose-lowering agents including metformin and insulin reduces the risk of albuminuria, but evidence is lacking that it reduces the risk of relevant clinical renal outcomes, such as doubling of the serum creatinine level, ESRD, or death from renal disease during the years of follow-up of the trials [82,83]. Of increasing interest, some new antidiabetic agents have demonstrated renoprotective effects, which occurred beyond improvement of glucose control [26,84] (Table 4).

\subsection{SGLT2 inhibitors}

SGLT2is exert their glucose-lowering effects by promoting glucosuria, an effect resulting also in body weight and fat mass reduction. Aside from these effects, they increase natriuresis and osmotic diuresis, thus reducing arterial blood pressure and plasma overload [85]. SGLT2 inhibitors certainly represent the most promising pharmacological class among glucose-lowering agents not only for cardiovascular but also renal protection in T2DM patients. Numerous excellent and extensive reviews were devoted to this topic in recent years, which summarized the preclinical and clinical data and provided several hypotheses to explain the nephroprotective effects of these new antidiabetic agents [18,19,21,86-90]. Effects of SGLT2is on the kidney are likely explained by multiple pathways, beyond the systemic effects via reductions in blood glucose, body weight and blood pressure. SGLT2is are associated with a reduction in glomerular hyperfiltration, an effect that is mediated through increased natriuresis and restored tubuloglomerular feedback, and independent of glycemic control (Figure 1). In addition, they may improve renal oxygenation and cellular energy metabolism [21] and also reduce intrarenal inflammation [91], thereby slowing the progression of kidney function decline.

Because of their specific mechanism of action targeting the kidney, SGLT2is lose part of their glucose-lowering activity when eGFR falls below $45-60 \mathrm{ml} / \mathrm{min} / 1.73 \mathrm{~m}^{2}$. Therefore, the use of SGLT2is is currently not recommended below this threshold [92,93], yet further studies may mitigate this restriction in the future. Indeed, blood pressure lowering effect of SGLT2is seems to be maintained [94,95] and a reduction in major cardiovascular events and mortality was reported in subgroups analyses of T2DM patients with eGFR below $60 \mathrm{ml} / \mathrm{min} / 1.73 \mathrm{~m}^{2}$ in cardiovascular outcome trials [96,97]. Even if SGLT2is consistently reduce systolic blood pressure [98], this specific effect seems to play a minor role in the improvement of renal outcomes in T2DM patients with wellcontrolled blood pressure at baseline [99].

In patients with T2DM at high cardiovascular risk recruited in EMPA-REG OUTCOME, empagliflozin was associated with slower progression of CKD, reflected by reduction in albuminuria and less decline in eGFR, and lower rates of clinically relevant renal events, including progression to ESRD, than was placebo when added to standard care (Table 4) [22]. A detailed post-hoc analysis supported short-term and longterm benefits of empagliflozin on UACR, irrespective of patients' albuminuria status at baseline [100]. Of note, the reductions in major cardiovascular events and mortality were also consistent across categories of eGFR and UACR at baseline [96]. 


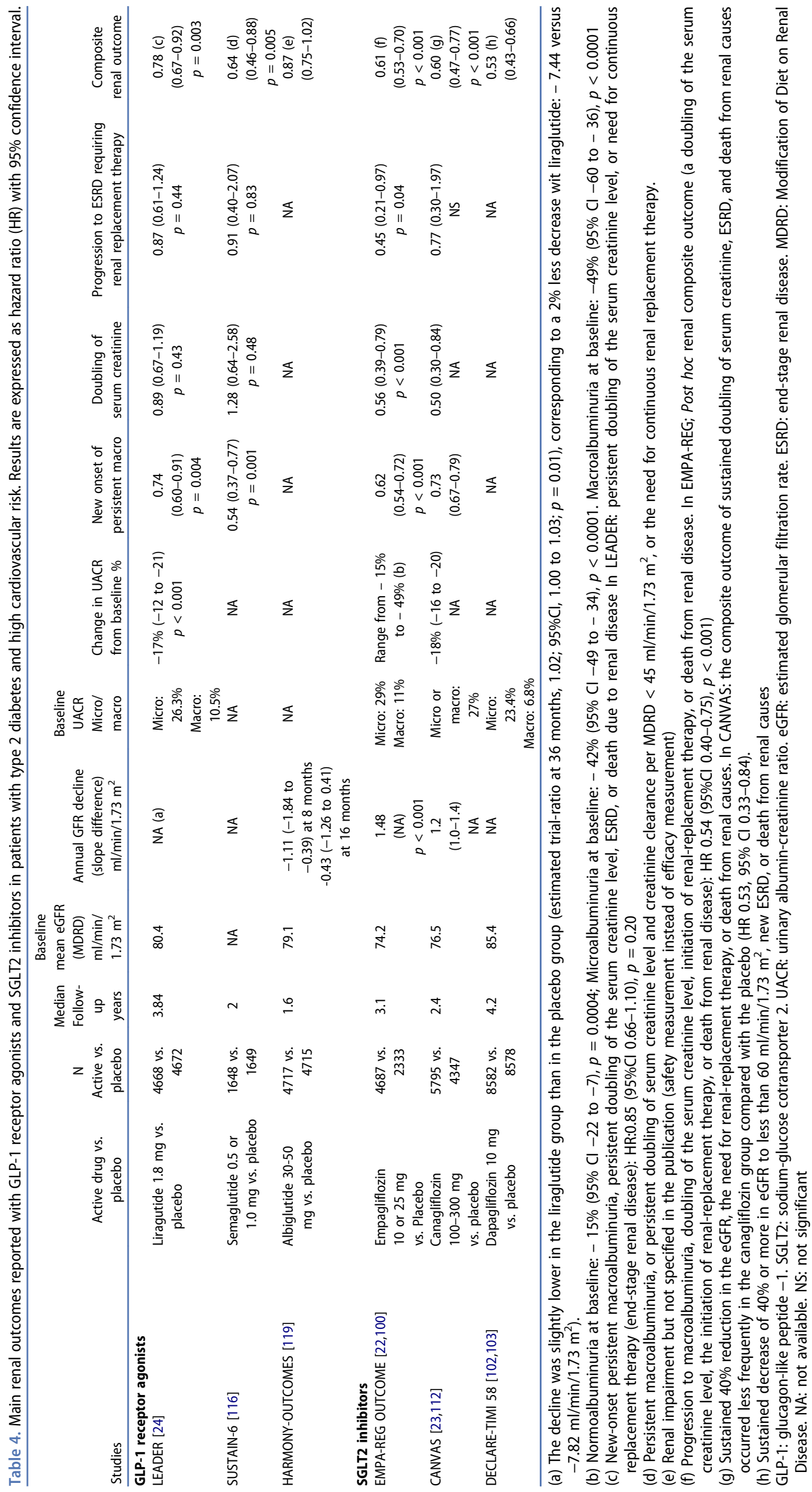


In the Canagliflozin Cardiovascular Assessment Study (CANVAS) Program, prespecified composite endpoint of sustained and adjudicated doubling in serum creatinine, ESRD, or death from renal causes occurred less frequently in the canagliflozin group compared with the placebo group, with consistent findings across prespecified patient subgroups [23]. Annual eGFR decline was slower and mean UACR was lower in participants treated with canagliflozin than in those treated with placebo. After a rather short median follow-up of 2.4 years, only a numerical trend for less progression to ESRD requiring renal replacement therapy was noticed (Table 4) [23]. Renal outcomes (HR, 0.59; 95\% $\mathrm{Cl}, 0.44-0.79$ versus $\mathrm{HR}, 0.63 ; 95 \% \mathrm{Cl}, 0.39-1.02$; interaction $p$ value $=0.73)$ were similarly reduced in the secondary and primary cardiovascular prevention cohorts of CANVAS, respectively [101]. Furthermore, relative effects on most cardiovascular and renal outcomes were similar across eGFR subgroups [97].

In the DECLARE ('Dapagliflozin Effect on CardiovascuLAR Events') TIMI 58 cardiovascular outcome trial [102], a prespecified renal event (including cardiovascular death) occurred in $4.3 \%$ in the dapagliflozin group and in $5.6 \%$ in the placebo group (HR 0.76; $95 \% \mathrm{Cl}, 0.67-0.87$ ) [103]. When the analysis excluded cardiovascular death (which was not affected by the treatment), the difference in the prespecified renal composite endpoint ( $40 \%$ decrease in eGFR, ESRD, or renal death) was even more important: HR 0.53 (0.43-0.66). No significant interaction $(p=0.87)$ was detected when the analysis was performed according to baseline eGFR levels, with less events in the dapagliglozin groups versus the placebo groups: $<60 \mathrm{ml} / \mathrm{min} / 1.73 \mathrm{~m}^{2}(n=606$ vs. $n=659$ ) HR 0.60 (0.35-1.02); eGFR 60 to $<90 \mathrm{ml} / \mathrm{min} / 1.73 \mathrm{~m}^{2}$ ( $n=3838$ vs. 3894): HR 0.54 (0.40-0.73); eGFR $\geq 90 \mathrm{ml} / \mathrm{min} / 1.73 \mathrm{~m}^{2}$ $(n=4137$ vs. $n=4025)$ : HR 0.50 (0.34-0.73). Acute kidney injury occurred less frequently in the dapagliflozin group than in the placebo group ( $1.5 \%$ vs. $2.0 \%$; HR 0.69 (0.55-0.87), $p=0.002$ ) [103]. Of note, in DECLARE-TIMI 58 , less patients with eGFR $<60 \mathrm{ml} / \mathrm{min} / 1.73 \mathrm{~m}^{2}(7.4 \%$, in fact a predefined exclusion criterion, but not always respected by the investigators) were recruited than in EMPA-REG OUTCOME (26\%) and CANVAS (20\%), which allowed recruitment of patients provided that eGFR was $>30 \mathrm{ml} / \mathrm{min} / 1.73 \mathrm{~m}^{2}$ ).

It is noteworthy that EMPA-REG OUTCOME, CANVAS, and DECLARE-TIMI 58 were cardiovascular outcome RCTs, with renal outcomes as secondary rather than primary endpoints, even if prespecified. According to a recent meta-analysis, the results regarding renal protection using the composite endpoint of renal worsening, ESRD, and renal death were remarkably consistent in the three trials and comparable in patients in secondary cardiovascular prevention (HR 0.56; 0.47-0.67) and primary cardiovascular prevention (HR 0.54; 0.42-0.71) [104]. The magnitude of benefit of SGLT2is varied with baseline renal function, with lesser reductions in progression of renal disease ( $p$ for interaction $=0.0258$ ) but greater reductions in hospitalizations for heart failure ( $p$ for interaction $=0.0073$ ) in patients with more severe kidney disease at baseline. These data should be confirmed in dedicated renal outcome trials such as three ongoing large prospective RCTs, CREDENCE
("Canagliflozin and Renal Endpoints in Diabetes with Established Nephropathy Clinical Evaluation [105] EMPAKIDNEY (The Study of Heart and Kidney Protection With Empagliflozin: Clinical Trials.gov Identifier: NCT03594110)and Dapa-CKD (A Study to Evaluate the Effect of Dapagliflozin on Renal Outcomes and Cardiovascular Mortality in Patients With Chronic Kidney Disease: ClinicalTrials.gov Identifier: NCT03036150).

Even if SGLT2is offer nephroprotection (together with cardioprotection), they may also be associated with adverse effects, including an increased risk of acute kidney injury [106], so that an individual benefit-risk balance should be taken into consideration $[107,108]$. Several mechanisms have been proposed to explain a risk of acute kidney injury with SGLT2is: effective volume depletion (dehydration, diuretic therapy), excessive decline in transglomerular pressure (concomitant RAAS blockade), induction of renal medullary hypoxic injury (for instance, triggered by nonsteroidal anti-inflammatory drugs) [108]. Combining these three conditions, which may act synergistically, would dramatically increase the risk of acute renal failure. A higher proportion of reports with acute renal failure were collected among reports with SGLT2is in the FDA adverse event report system (FAERS) database [109]. As a consequence, the Food and Drug Administration (FDA) now requires that acute kidney injury be listed as a potential side effect of the SGLT2is along with cautious prescription of these glucoselowering agents with other medications, such as RAAS antagonists, diuretics, and nonsteroidal anti-inflammatory drugs. It is imperative to ascertain whether the reported acute renal failure represents true structural kidney injury or only a functional decline in glomerular filtration rate [110]. Of note, the recent data of the DECLARE-TIMI 58 trial are reassuring to this respect, with less cases of renal impairment in the dapagliflozin group than in the placebo group, despite $81.3 \%$ of patients were treated with RAAS inhibitors at baseline [103].

Another dreaded adverse event that has been associated with SGLT2 use is lower-limb amputation. In a recent extensive systematic review, there are strong overall associations of SGLT2 inhibition with protection against serious decline in kidney function (RR 0.59, 95\% Cl 0.49-0.71), besides major cardiovascular events, heart failure and all-cause death. However, SGLT2 inhibitors were associated with amputations ( $\mathrm{RR} 1.44,95 \% \mathrm{Cl} 1.13-1.83$ ), aside of wellknown adverse events such as genital infections and volume depletion effects [111]. According to this review, this association with amputation appears to differ between individual SGLT2 inhibitors, with a higher risk for canagliflozin [111], a difference mainly driven by the results of CANVAS [112]. This increased risk of lower limb amputation was also observed in some observational studies and pharmacovigilance reports, yet whether it is a class effect or not remains an open question [113]. Of note, patients with ESRD who receive dialysis are at high risk of lower extremity amputation, which is associated with a high mortality rate, and among those people with ESRD, patients with diabetes had amputation rates more than five times as high as patients without diabetes [114].

\subsection{GLP-1 receptor agonists}

GLP-1RAs act on traditional risk factors for progressive kidney disease including improved glucose control, blood pressure 
lowering, insulin-sparing effect and body weight reduction. Furthermore, GLP-1RAs can also have direct effects in the kidney (review in [25] and [115]). However, the mechanisms that may underlie any direct actions in the kidney remain to be established and might be multiple: effects on the intrarenal RAAS, ischemia/hypoxia, apoptosis, and neural signaling. Furthermore, GLP-1RAs have also been shown to reduce inflammation, macrophage infiltration, oxidative stress, and the accumulation of type 4 collagen in the kidney $[25,115]$. The GLP-1 receptor is expressed in glomeruli and arterioles, yet kidney protective actions independent of the GLP-1 receptor have also been proposed. GLP-1 induces natriuresis by reducing $\mathrm{Na} / \mathrm{H}$ exchange transporter isoform 3-dependent sodium reabsorption in the proximal tubule [25].

In cardiovascular outcome trial LEADER, the prespecified secondary renal outcome was a composite of new-onset persistent macroalbuminuria, persistent doubling of the serum creatinine level, end-stage renal disease, or death due to renal disease [24]. After a median follow-up of 3.84 years in T2DM patients at high risk of cardiovascular disease, this renal outcome occurred in fewer participants in the liraglutide group than in the placebo group (HR 0.78; 95\% Cl, 0.67-0.92; $p=0.003$ ) (Table 4). This result was driven primarily by the new onset of persistent macroalbuminuria, whereas no significant differences were noticed for persistent doubling of the serum creatinine level, end-stage renal disease, or death due to renal disease (Table 4). The rates of renal adverse events were similar in the liraglutide group and the placebo group (15.1 events and 16.5 events per 1000 patient-years), including the rate of acute kidney injury (7.1 and 6.2 events per 1000 patient-years, respectively) [24].

In SUSTAIN-6, after a median follow-up of 2 years, new or worsening nephropathy occurred less frequently in T2DM patients treated with semaglutide $(\mathrm{HR}=0.64 ; 95 \% \mathrm{Cl} 0.46-$ $0.88 ; p<0.01)$. However, as in LEADER, this composite outcome was largely driven by a reduction in new-onset macroalbuminuria, whereas doubling of serum creatinine concentration to an eGFR $\leq 45 \mathrm{ml} / \mathrm{min}$ per $1.73 \mathrm{~m}^{2}$, ESRD, and death of renal cause were unaffected (Table 4) [116]. In EXSCEL, a reduction of new-onset macroalbuminuria ( $2.2 \%$ vs. $2.8 \% ; P=0.03$ ) was also reported in patients treated with once weekly exenatide compared with placebo, without significant changes neither in microalbuminuria (7.2 vs. 7.5\%) nor in ESRD requiring replacement therapy $(0.7$ vs. $0.9 \%)$ after a median follow-up of 3.2 years [117]. ELIXA recruited T2DM patients who had had a recent acute coronary event and a eGFR > $30 \mathrm{ml} / \mathrm{min} / 1.73 \mathrm{~m}^{2}$ (mean value 76) [118]. Baseline characteristics showed that $74.3 \%$ of patients had normoalbuminuria, $19.2 \%$ microalbuminuria and $6.5 \%$ macroalbuminuria. A modest reduction in the increase in albuminuria was observed after 24-month treatment with lixisenatide compared with placebo $(+24 \%$ vs. $+34 \%, p=0.004$, but $p=0.07$ after adjustment for HbA1c) [118]. In HARMONYOUTCOMES, T2DM patients with established cardiovascular disease treated with albiglutide showed significantly less cardiovascular complications than those treated with placebo [119]. Around $19 \%$ of patients had diabetic nephropathy at baseline, yet mean eGFR averaged $79 \mathrm{ml} / \mathrm{min} / 1.73 \mathrm{~m}^{2}$ and no data on albuminuria were reported. A slight reduction in eGFR was noticed in the albiglutide group compared with the placebo group, significant at 8 months but not anymore at 16 months (Table 4). Renal events were not analyzed as an efficacy but a safety outcome. Renal impairment (not precisely defined) trended to occur less frequently in patients treated with albiglutide than in patients having received placebo (6 vs. 7\%) (Table 4).

Integrated data from 9 phase 2 and 3 trials in T2DM patients ( $N=6005)$ showed that dulaglutide did not affect eGFR, but slightly decreased UACR, without increasing kidney adverse events, when compared to placebo or active comparators [120]. In the 52-week AWARD-7 trial in patients with T2DM and moderate-to-severe CKD, once-weekly dulaglutide produced glycemic control similar to that achieved with insulin glargine, no greater reduction in $\mathrm{UACR}$, but significantly reduced decline in eGFR ( $p=0.005$ for dulgalutide $1.5 \mathrm{mg}$ and $p=0.009$ for dulaglutide $0.75 \mathrm{mg}$ vs. insulin) [121]. Overall these short-term data suggest that dulaglutide has the potential to exert renal protection in patients with T2DM, an effect that should be confirm in long-term studies with clinical hard endpoints [122].

Divergent results were reported in recent meta-analyses that investigated the effects of GLP-1RAs on microvascular and renal complications [123-125]. Thus, even if most studies report beneficial effects of GLP-1RAs on the incidence of albuminuria, more evidence is needed to convincingly support positive effects on renal function and progression to ESRD in patients with T2DM.

\section{Other pharmacological agents in development}

Considering the complex pathophysiology of CKD in T2DM and the residual renal risk despite RAAS inhibition, even when some new glucose-lowering agents with proven renoprotective properties are added, there is still room for innovative strategies. Whereas classical approaches targeted intrarenal hemodynamic abnormalities, new approaches focus on the potential to reduce fibrosis, low-grade inflammation, oxidative stress, advanced glycation end products (AGE), all processes involved in renal failure in T2DM [22]. Table 5 summarizes new therapies tested in humans at different stages of development and in-depth reviews on this topic may be found elsewhere $[8,126-130]$. In phase 2 trials, most agents showed their ability to reduce albuminuria and may also exert some protection on eGFR decline, but these effects were not yet confirmed in phase 3 trials for many of them. Of note, some promising drugs tested in large RCTs gave negative results or were associated with adverse events [131]. Globally, confirming safety remains a priority before use in clinical practice. Some concerns exist, for example, with endothelin receptor antagonist (fluid overload and heart failure, especially with avosentan whose development has been stopped, whereas the efficacy and safety of another compound atrasentan is currently investigated in the ongoing SONAR trial) [132], or bardoxolone (more cardiovascular events and worse albuminuria) [131]. Overall, for all these pharmacological interventions, the nephroprotective efficacy was mainly speculative and remains largely unproven. 


\begin{tabular}{|c|c|}
\hline \multicolumn{2}{|l|}{ Anti-fibrotic effects } \\
\hline PKC (protein kinase C) inhibitors & Ruboxistaurin \\
\hline Glycosaminoglycans & Sulodexide \\
\hline Anti-TGF (transforming growth factor) & FG-3019, pirfenidone, LY2382770, LY3016859 \\
\hline Metalloprotease inhibitors & XL 784, doxycycline, minocycline \\
\hline Serotonin receptor antagonists & Sarpogrelate \\
\hline \multicolumn{2}{|l|}{ Anti-inflammatory effects } \\
\hline Phosphodiesterase inhibitors & Pentoxifylline, CPT-499, PF-00489791 \\
\hline Janus kinase inhibitors & Baricitinib \\
\hline Endothelin receptor antagonists & Avosentan, atrasentan, dagutril \\
\hline \multirow[t]{2}{*}{ Chemokine inhibitors } & CCX140-13, PF-04634817, BMS-813160 \\
\hline & Bindarit, Emapticap pegol \\
\hline \multicolumn{2}{|l|}{ Anti-oxidant effects } \\
\hline Activation of Nrf2 pathway & Bardoxolone \\
\hline Inhibitors of NADPH oxidase 1 and 4 & GKT-137831 \\
\hline a-Lipoic acid (+ losartan) & INV-144 \\
\hline Reduction of oxidative stress, reduction of Ox-LDL & Probucol \\
\hline Pleiotropic effects, activation of Nrf2 pathway & Silymarin \\
\hline Pleiotropic effects of polyphenol & Resveratrol \\
\hline \multicolumn{2}{|l|}{ AGEs inhibitors } \\
\hline & Pyridoxamine, pimagedine, TTP448 \\
\hline \multicolumn{2}{|l|}{ Vitamin D activators } \\
\hline Multiple effects & Cholecalciferol, paricalcitol, calcitriol, and a-calcidol \\
\hline
\end{tabular}

\section{Conclusions}

Diabetic kidney disease becomes increasingly prevalent among patients with T2DM and is associated with increased mortality, decreased quality of life and high healthcare cost. Thus preventing early CKD in T2DM patients and limiting the progression toward ESRD are major challenges in clinical practice. Besides healthy lifestyle and the avoidance of nephrotoxic agents, it is essential to maintain adequate control of blood glucose and blood pressure as both chronic hyperglycemia and hypertension exert deleterious effects on kidney structure and function. RAAS inhibitors were the first drugs that have proven their efficacy to prevent surrogate and hard clinical renal outcomes, independently of their antihypertensive effects. Their ability to control albuminuria/proteinuria beyond the reduction of blood pressure appears of major importance. In all guidelines, they are recommended in T2DM patients with any stage of CKD, from very early stage to more advanced disease, that is 3 to 4 stages CKD, especially if (micro)albuminuria is present. Overall, ACE inhibitors and ARA II are considered to exert equal renoprotective effects.

New glucose-lowering agents, GLP-1RAs and especially SGLT2is, have recently shown nephroprotective properties on composite renal outcomes combining surrogate endpoints (albuminuria, eGFR decline) and hard clinical events (progression to ESRD requiring renal replacement therapies). These effects were observed when added to standard care, including RAAS blockers, in T2DM patients with established or at high risk of cardiovascular disease. The nephroprotection by SGLT2is occurs independently of glucose control and this observation opens new perspectives for the treatment on nondiabetic CKD currently in investigation. Whether SGLT2is also exert nephroprotection in T2DM patients with more advanced stages of the disease (stages 3b-4 CKD) remains to be carefully investigated in dedicated studies with renal outcomes as primary endpoints, as well as the complex and pleiotropic underlying mechanisms contributing to renoprotection.

Finally, despite the favorable effects of these pharmacological approaches, even if combined, the renal residual risk remains high in many T2DM patients. Therefore, new therapies are in development targeting fibrosis, low-grade inflammation, oxidative stress or AGE deposition, all processes involved in progressive renal impairment in T2DM. However, despite some promising results, all these new drugs first have to prove both their efficacy and safety in large RCTs before any future use in clinical practice.

\section{Expert opinion}

In parallel with the T2DM pandemic, CKD related to diabetes has become the leading cause of ESRD in many countries worldwide, and is associated with high cardiovascular morbidity and mortality. Based on landmark clinical trials, diabetes-related CKD is preventable by controlling conventional factors, mainly hyperglycemia and hypertension, with multifactorial therapies combining lifestyle and drug interventions. Many antidiabetic medications (metformin, glitazones, DPP-4 inhibitors) have shown promising results in animal models of CKD and offered the possibility to investigate potential molecular mechanisms that may explain renoprotection. However, although useful, these animal models are not perfect [133], so that preclinical data should be confirmed in well-designed clinical studies. For all these antidiabetic agents, only effects on biomarkers and soft surrogate endpoints such as reduction of albuminuria and more rarely reduction in eGFR have been reported [83]. 
A biomarker is an objectively measured characteristic that is indicative of some underlying phenomenon or process (i.e. albuminuria), while a surrogate is a biomarker that 'takes the place' of a clinically meaningful outcome, usually earlier in the disease process (i.e. reduction in eGFR) [134]. Even, if changes in albuminuria and eGFR were associated with a greater risk of experiencing clinical outcomes [135], clinicians are waiting for clear-cut data on more relevant hard endpoints, such as avoiding the progression to ESRD or renal death. Some well-known agents (RAAS inhibitors) and, more recently, new antidiabetic medications (SGLT2is) have shown renoprotective effects, not only on surrogate endpoints but also on hard clinical outcomes, independently of their blood pressure-lowering activity and glucose-lowering activity, respectively. However, even if combined therapy is used, the remaining risk of CKD progression in T2DM patients is still high.

Direct comparison of agents within a pharmacologic class or between drug classes is hazardous, as renal outcomes are inconsistently defined across trials. As a consequence, the impact of many specific drugs on renal outcome measures in patients with T2DM remains unclear. The evaluation of hard clinical outcomes such as progression to ESRD or death of renal cause requires a long follow-up in large cohorts. As it is hard to carry out and finance such studies, most recent publications use composite renal outcomes, a mix of surrogate and hard endpoints, which, however, may differ across RCTs (Table 1-4). Of note, these composite endpoints can reveal significant results driven by a single surrogate marker, for instance reduction in albuminuria, but not clinical events of true relevance to T2DM patients, for instance need for renal replacement therapy. Renal outcome studies including a welldefined, standardized core set of patient-relevant outcomes are needed to achieve evidence-based guidance and improve clinical care for T2DM patients at risk of CKD [136,137]. A doubling of serum creatinine level is generally used in most studies either as individual endpoint or as part of a composite outcome. However, it is a rather late event in CKD natural history, corresponding to a change in eGFR of more than $50 \%$. There is great interest in considering alternative endpoints for clinical trials to shorten their duration, reduce sample size, and extend their conduct to patients with earlier stages of CKD. A 30\% declines in eGFR over 2 years occurred more commonly than a doubling of serum creatinine concentration and was strongly and consistently associated with the risk of ESRD and mortality [138]. A scientific workshop sponsored by the National Kidney Foundation and the US FDA concluded that a confirmed decline in eGFR of $30 \%$ over 23 years may be a valuable alternative surrogate end point for CKD progression in most circumstances [139]. Another challenge is to improve clinical trial enrolment criterion to identify T2DM patients at higher risk of ESRD [140]. This improvement will be crucial to reduce the sample size and ameliorate the statistical power by increasing the number of key events in RCTs. It will also be of major importance in clinical practice to more effectively select T2DM patients who should require intensive combining therapy for a better renal protection.

RAAS inhibitors and SGLT2 inhibitors are the two pharmacologic classes that have best proven to improve both surrogate and hard clinical endpoints in patients with T2DM. RAAS inhibitors were initially developed as antihypertensive agents, yet they renoprotective effects appear to be at least partially independent of their blood pressure lowering effects. These agents also reduce proteinuria, a risk marker for renal disease progression [70] and accumulating evidence indicates that their antiproteinuric effect correlates with their additional renal benefits [71]. High dosing of ACE inhibitors or ARA II may be a valuable approach for providing greater reduction in proteinuria and ultimately renoprotection [72]. Similarly, SGLT2 inhibitors were developed as antihyperglycemic agents, yet their nephroprotection is essentially independent of their glucose-lowering effects. Both pharmacological classes modify intrarenal hemodynamic properties by complementary mechanisms contributing to reduce intraglomerular pressure $[141,142]$ (Figure 1). RAAS inhibitors, by inhibiting vasoconstrictive effects of angiotensin 2, dilate postglomerular artery whereas SGLT2 inhibitors, by restoring tubuloglomerular feedback, constrict preglomerular artery. Intriguingly, experimental studies in rats suggested that dapagliflozin-associated beneficial effects on diabetic nephropathy might result from suppression of renal RAAS component expression, contributing to reduction in oxidative stress and interstitial fibrosis [143]. In hypertensive patients with T2DM on stable RAAS blocker therapy, dapagliflozin significantly reduced albuminuria, a reduction that remained present after adjusting for changes in $\mathrm{HbA1C}$, systolic blood pressure, body weight and eGFR [144]. However, T2DM patients who did not respond to RAAS inhibition by a reduction in albuminuria also did not respond to dapagliflozin, indicating that individual therapy resistance to RAAS inhibition cannot be overcome with the addition of SGLT2 inhibitors. These data suggest that the individual drug resistance may be an intrinsic individual characteristic unrelated to the type of pharmacologic intervention, unless the mode of action of the SGLT2 inhibitor on albuminuria is through the RAAS [145]. Of note, in both EMPA-REG OUTCOME and CANVAS, almost three-quarters of T2DM patients were treated with RAAS inhibitors at baseline and throughout the trials. Subgroup analyses showed no significant interaction between users and non-users of RAAS blockers regarding primary cardiovascular composite endpoints and cardiovascular death both in EMPA-REG OUTCOME [146] and in CANVAS [112]. No such subanalysis is reported in DECLARETIMI 58 [103] neither for renal outcomes yet. Both RAAS inhibitors and SGLT2 inhibitors are renoprotective, but may also exert deleterious effects leading to acute kidney insufficiency in certain circumstances, such as dehydration and association with nonsteroidal anti-inflammatory agents [106,110,147]. As a consequence, caution and appropriate supervision should be recommended when using these agents in more frailty patients, for instance elderly patients. Whereas RAAS blockers may induce hyperkalemia, as previous discussed, SGLT2 inhibitors are generally not associated with ionic disturbances.

The effects of empagliflozin [96] and canagliflozin [97] on cardiovascular and renal outcomes were not modified by baseline level of kidney function in T2DM patients with a history or high risk of cardiovascular disease down to eGFR levels of $30 \mathrm{~mL} /$ $\mathrm{min} / 1.73 \mathrm{~m}^{2}$. Thus, reassessing current limitations on the use of SGLT2 inhibitors in patients with CKD at high risk of cardiovascular disease may allow additional individuals to benefit from 
this therapy. Furthermore, the glucose-independent hemodynamic intrarenal mechanisms of SGLT2 inhibitors provide the possibility to extend the use of these medications to nondiabetic kidney disease. This should be confirmed in ongoing dedicated trials. If results were positive, they will have the potential to change clinical practice and outlook of high-risk patients with diabetic and nondiabetic CKD.

Because of the beneficial effects of SGLT2 inhibitors not only on surrogate endpoints but also on hard renal outcomes (in contrast to what has been shown with GLP-1RAs) [148], the recent consensus report by the American Diabetes Association (ADA) and the European Association for the Study of Diabetes (EASD) considers that SGLT2 inhibitors with evidence of reducing CKD in cardiovascular outcome trials should be considered as the best pharmacological option to be added to metformin in T2DM patients with CKD, provided that eGFR is adequate. If SGLT2 inhibitors are not tolerated or contraindicated or if eGFR is less than adequate, the addition of a GLP1RA with proven cardiovascular benefit may be considered [149].

The economic burden of progressive CKD among T2DM patients is high [150]. Whether new pharmacological approaches currently in development will succeed to reduce the residual risk of CKD in T2DM patients remains an open question. The future challenge will be to find combined pharmacological therapies offering the best renal (and cardiovascular) protection, with a good safety profile and at a reasonable cost.

\section{Funding}

This manuscript was not funded.

\section{Declaration of interest}

AJ Scheen has received lecture/advisor fees from AstraZeneca, Boehringer Ingelheim, Eli Lilly and Company, Janssen Pharmaceuticals, Merck Sharp \& Dohme, Novartis, Novo Nordisk, Sanofi and Servier. The authors have no other relevant affiliations or financial involvement with any organization or entity with a financial interest in or financial conflict with the subject matter or materials discussed in the manuscript apart from those disclosed.

\section{Reviewer Disclosures}

Peer reviewers on this manuscript have no relevant financial or other relationships to disclose.

\section{References}

Papers of special note have been highlighted as either of interest $(\cdot)$ or of considerable interest (..) to readers.

1. Tuttle KR, Bakris GL, Bilous RW, et al. Diabetic kidney disease: a report from an ADA consensus conference. Am J Kidney Dis. 2014;64:510-533.

2. Muskiet MH, Tonneijck L, Smits MM, et al. Pleiotropic effects of type 2 diabetes management strategies on renal risk factors. Lancet Diabetes Endocrinol. 2015;3:367-381.

3. Anders HJ, Huber TB, Isermann B, et al. CKD in diabetes: diabetic kidney disease versus nondiabetic kidney disease. Nat Rev Nephrol. 2018;14:361-377.

4. Xie Y, Bowe B, Mokdad AH, et al. Analysis of the global burden of disease study highlights the global, regional, and national trends of chronic kidney disease epidemiology from 1990 to 2016. Kidney Int. 2018;94:567-581.

5. Koye DN, Shaw JE, Reid CM, et al. Incidence of chronic kidney disease among people with diabetes: a systematic review of observational studies. Diabet Med. 2017;34:887-901.

6. Thomas MC, Cooper ME, Zimmet P. Changing epidemiology of type 2 diabetes mellitus and associated chronic kidney disease. Nat Rev Nephrol. 2016;12:73-81.

7. Koye DN, Magliano DJ, Nelson RG, et al. The global epidemiology of diabetes and kidney disease. Adv Chronic Kidney Dis. 2018;25:121-132.

8. Alicic RZ, Rooney MT, Tuttle KR. Diabetic kidney disease: challenges, progress, and possibilities. Clin J Am Soc Nephrol. 2017;12:2032-2045.

9. American Diabetes Association. 10. Microvascular complications and foot care: standards of medical care in diabetes-2018. Diabetes Care. 2018;41:S105-S18.

10. Maclsaac RJ, Jerums G, Ekinci El. Glycemic control as primary prevention for diabetic kidney disease. Adv Chronic Kidney Dis. 2018;25:141-148.

11. Ruospo M, Saglimbene VM, Palmer SC, et al. Glucose targets for preventing diabetic kidney disease and its progression. Cochrane Database Syst Rev. 2017;6:CD010137.

12. Scheen AJ. Pharmacokinetic considerations for the treatment of diabetes in patients with chronic kidney disease. Expert Opin Drug Metab Toxicol. 2013;9:529-550.

13. Neumiller JJ, Alicic RZ, Tuttle KR. Therapeutic considerations for antihyperglycemic agents in diabetic kidney disease. J Am Soc Nephrol. 2017;28:2263-2274.

14. de Galan BE, Perkovic V, Ninomiya T, et al. Lowering blood pressure reduces renal events in type 2 diabetes. J Am Soc Nephrol. 2009;20:883-892.

15. Zoungas S, de Galan BE, Ninomiya $T$, et al. Combined effects of routine blood pressure lowering and intensive glucose control on macrovascular and microvascular outcomes in patients with type 2 diabetes: new results from the ADVANCE trial. Diabetes Care. 2009;32:2068-2074.

16. Patney V, Chaudhary K, Whaley-Connell A. Treatment of diabetic kidney disease with hypertension control and renin angiotensin system inhibition. Adv Chronic Kidney Dis. 2018;25:158-165.

17. Palmer SC, Mavridis D, Navarese E, et al. Comparative efficacy and safety of blood pressure-lowering agents in adults with diabetes and kidney disease: a network meta-analysis. Lancet. 2015;385:2047-2056.

18. Fioretto $P$, Zambon A, Rossato $M$, et al. SGLT2 inhibitors and the diabetic kidney. Diabetes Care. 2016;39(Suppl 2):S165-71.

19. Nespoux J, Vallon V. SGLT2 inhibition and kidney protection. Clin Sci (Lond). 2018;132:1329-1339.

20. Alicic RZ, Johnson EJ, Tuttle KR. SGLT2 inhibition for the prevention and treatment of diabetic kidney disease: a review. Am J Kidney Dis. 2018;72:267-277.

21. Heerspink HJL, Kosiborod M, Inzucchi SE, et al. Renoprotective effects of sodium-glucose cotransporter-2 inhibitors. Kidney Int. 2018;94:26-39.

22. Wanner C, Inzucchi SE, Lachin JM, et al. Empagliflozin and progression of kidney disease in type 2 diabetes. $N$ Engl J Med. 2016;375:323-334.

.. Large prospective trial (EMPA-REG OUTCOME) demonstrating that empagliflozin is associated with slower progression of kidney disease and lower rates of clinically relevant renal events than was placebo when added to standard care in patients with type 2 diabetes at high cardiovascular risk.

23. Perkovic V, Zeeuw D, Mahaffey KW, et al. Canagliflozin and renal outcomes in type 2 diabetes: results from the CANVAS Program randomised clinical trials. Lancet Diabetes Endocrinol. 2018;6:691-704.

In a prespecified exploratory analysis of the CANVAS program, canagliflozin treatment was associated with a reduced risk of sustained loss of kidney function, attenuated eGFR decline, and a reduction in albuminuria, which supports a possible renoprotective effect of this drug in people with type 2 diabetes. 
24. Mann JFE, Orsted DD, Brown-Frandsen K, et al. Liraglutide and renal outcomes in type 2 diabetes. N Engl J Med. 2017; 377(839-48)

-. Large prospective trial (LEADER) showing that, when added to usual care, liraglutide resulted in lower rates of the development and progression of diabetic kidney disease than placebo in patients with T2DM and high cardiovascular risk.

25. Thomas MC. The potential and pitfalls of GLP-1 receptor agonists for renal protection in type 2 diabetes. Diabetes Metab. 2017;43 (Suppl 1):2S20-2S27.

26. Davies $M$, Chatterjee $S$, Khunti K. The treatment of type 2 diabetes in the presence of renal impairment: what we should know about newer therapies. Clin Pharmacol. 2016;8:61-81.

27. Goldenberg RM, Berall M, Chan CTM, et al. Managing the course of diabetic kidney disease: from the old to the new. Can J Diabetes. 2018;42:325-334.

28. Pickering RJ, Rosado CJ, Sharma A, et al. Recent novel approaches to limit oxidative stress and inflammation in diabetic complications. Clin Transl Immunology. 2018;7:e1016.

29. Magee C, Grieve DJ, Watson CJ, et al. Diabetic nephropathy: a tangled web to unweave. Cardiovasc Drugs Ther. 2017;31:579-592.

30. Emdin CA, Rahimi K, Neal B, et al. Blood pressure lowering in type 2 diabetes: a systematic review and meta-analysis. JAMA. 2015;313:603-615.

31. Brunstrom M, Carlberg B. Effect of antihypertensive treatment at different blood pressure levels in patients with diabetes mellitus: systematic review and meta-analyses. BMJ. 2016;352:i717.

32. Kidney Disease: improving Global Outcomes (KDIGO) Blood Pressure Work Group. KDIGO clinical practice guideline for the management of blood pressure in chronic kidney disease. Kidney Int. 2012;2:337-414.

33. Whelton PK, Carey RM, Aronow WS, et al. 2017 ACC/AHA/AAPA/ ABC/ACPM/AGS/APhA/ASH/ASPC/NMA/pcna guideline for the prevention, detection, evaluation, and management of high blood pressure in adults: a report of the American college of cardiology/american heart association task force on clinical practice guidelines. Hypertension. 2018;71:e13-e115.

34. Thomopoulos C, Parati G, Zanchetti A. Effects of blood-pressurelowering treatment on outcome incidence in hypertension: 10 should blood pressure management differ in hypertensive patients with and without diabetes mellitus? Overview and meta-analyses of randomized trials. J Hypertens. 2017;35:922-944.

35. Guideline development group. Clinical practice guideline on management of patients with diabetes and chronic kidney disease stage $3 \mathrm{~b}$ or higher (eGFR $<45 \mathrm{~mL} / \mathrm{min}$ ). Nephrol Dial Transplant. 2015;30(Suppl 2):ii1-142.

36. Lamprea-Montealegre $\mathrm{JA}$, de Boer $\mathrm{IH}$. Reevaluating the evidence for blood pressure targets in type 2 diabetes. Diabetes Care. 2018;41:1132-1133.

37. Lewis EJ, Hunsicker LG, Clarke WR, et al. Renoprotective effect of the angiotensin-receptor antagonist irbesartan in patients with nephropathy due to type 2 diabetes. N Engl J Med. 2001;345:851-860.

-. Large clinical trial showing benefits on hard renal endpoint for RAAS inhibitors (ARA II irbesartan) in type 2 diabetic patients with CKD and albuminuria.

38. Brenner BM, Cooper ME, de Zeeuw D, et al. Effects of losartan on renal and cardiovascular outcomes in patients with type 2 diabetes and nephropathy. N Engl J Med. 2001;345:861-869.

- Large clinical trial showing benefits on hard renal endpoint for RAAS inhibitors (ARA II losartan) in type 2 diabetic patients with CKD and albuminuria.

39. Heart Outcomes Prevention Evaluation Study Investigators. Effects of ramipril on cardiovascular and microvascular outcomes in people with diabetes mellitus: results of the HOPE study and MICRO-HOPE substudy. Lancet. 2000;355:253-259.

40. Patel A, MacMahon S, Chalmers J, et al. Effects of a fixed combination of perindopril and indapamide on macrovascular and microvascular outcomes in patients with type 2 diabetes mellitus (the ADVANCE trial): a randomised controlled trial. Lancet. 2007;370:829-840.

41. Anderson S, Brenner BM. Intraglomerular hypertension: implications and drug treatment. Annu Rev Med. 1988;39:243-253.
42. Roscioni SS, Heerspink HJ, de Zeeuw D. The effect of RAAS blockade on the progression of diabetic nephropathy. Nat Rev Nephrol. 2014; 10:77-87.

43. Ruiz-Ortega $M$, Ruperez $M$, Esteban V, et al. Angiotensin II: a key factor in the inflammatory and fibrotic response in kidney diseases. Nephrol Dial Transplant. 2006;21:16-20.

44. UK Prospective Diabetes Study Group. Efficacy of atenolol and captopril in reducing risk of macrovascular and microvascular complications in type 2 diabetes: UKPDS 39. BMJ. 1998;317:713-720.

45. Rahman M, Pressel S, Davis BR, et al. Renal outcomes in high-risk hypertensive patients treated with an angiotensin-converting enzyme inhibitor or a calcium channel blocker vs a diuretic: a report from the Antihypertensive and Lipid-Lowering Treatment to Prevent Heart Attack Trial (ALLHAT). Arch Intern Med. 2005;165:936-946.

46. Rahman $M$, Ford $C E$, Cutler JA, et al. Long-term renal and cardiovascular outcomes in Antihypertensive and Lipid-Lowering Treatment to Prevent Heart Attack Trial (ALLHAT) participants by baseline estimated GFR. Clin J Am Soc Nephrol. 2012;7:989-1002.

47. Scheen AJ. Type 2 diabetes and thiazide diuretics. Curr Diab Rep. 2018;18:6.

48. Marre M, Lievre M, Chatellier G, et al. Effects of low dose ramipril on cardiovascular and renal outcomes in patients with type 2 diabetes and raised excretion of urinary albumin: randomised, double blind, placebo controlled trial (the DIABHYCAR study). BMJ. 2004;328:495.

49. Daly CA, Fox KM, Remme WJ, et al. The effect of perindopril on cardiovascular morbidity and mortality in patients with diabetes in the EUROPA study: results from the PERSUADE substudy. Eur Heart J. 2005;26:1369-1378.

50. Catala-Lopez F, Macias Saint-Gerons D, Gonzalez-Bermejo D, et al. Cardiovascular and renal outcomes of renin-angiotensin system blockade in adult patients with diabetes mellitus: a systematic review with network meta-analyses. PLoS Med. 2016;13:e1001971.

- A network meta-analysis on the effect of RAAS inhibitors versus placebo or other therapies on renal outcomes in diabetic patients.

51. Vejakama P, Thakkinstian A, Lertrattananon D, et al. Renoprotective effects of renin-angiotensin system blockade in type 2 diabetic patients: a systematic review and network meta-analysis. Diabetologia. 2012;55:566-578.

52. Wu HY, Huang JW, Lin HJ, et al. Comparative effectiveness of renin-angiotensin system blockers and other antihypertensive drugs in patients with diabetes: systematic review and bayesian network meta-analysis. BMJ. 2013;347:f6008.

53. Persson F, Lindhardt $M$, Rossing $P$, et al. Prevention of microalbuminuria using early intervention with renin-angiotensin system inhibitors in patients with type 2 diabetes: A systematic review. J Renin Angiotensin Aldosterone Syst. 2016;17(3). pii: 1470320316652047.

54. Haller $\mathrm{H}$, Ito S, Izzo JL Jr., et al. Olmesartan for the delay or prevention of microalbuminuria in type 2 diabetes. $\mathrm{N}$ Engl J Med. 2011;364:907-917.

55. Nakao K, Hirata M, Oba K, et al. Role of diabetes and obesity in outcomes of the candesartan antihypertensive survival evaluation in Japan (CASE-J) trial. Hypertens Res. 2010;33:600-606.

56. Barnett AH, Bain SC, Bouter $P$, et al. Angiotensin-receptor blockade versus converting-enzyme inhibition in type 2 diabetes and nephropathy. N Engl J Med. 2004;351:1952-1961.

57. Mann JF, Schmieder RE, McQueen M, et al. Renal outcomes with telmisartan, ramipril, or both, in people at high vascular risk (the ONTARGET study): a multicentre, randomised, double-blind, controlled trial. Lancet. 2008;372:547-553.

58. Strippoli GF, Craig M, Deeks JJ, et al. Effects of angiotensin converting enzyme inhibitors and angiotensin II receptor antagonists on mortality and renal outcomes in diabetic nephropathy: systematic review. BMJ. 2004;329:828.

59. Kunz R, Friedrich C, Wolbers M, et al. Meta-analysis: effect of monotherapy and combination therapy with inhibitors of the renin angiotensin system on proteinuria in renal disease. Ann Intern Med. 2008;148:30-48.

60. van der Sande NG, Dorresteijn JA, Visseren FL, et al. Individualized prediction of the effect of angiotensin receptor blockade on renal 
and cardiovascular outcomes in patients with diabetic nephropathy. Diabetes Obes Metab. 2016;18:1120-1127.

61. Imai E, Chan JC, Ito $S$, et al. Effects of olmesartan on renal and cardiovascular outcomes in type 2 diabetes with overt nephropathy: a multicentre, randomised, placebo-controlled study. Diabetologia. 2011;54:2978-2986.

62. Fried LF, Emanuele N, Zhang JH, et al. Combined angiotensin inhibition for the treatment of diabetic nephropathy. $\mathrm{N}$ Engl J Med. 2013;369:1892-1903.

63. Parving HH, Brenner BM, McMurray JJ, et al. Cardiorenal end points in a trial of aliskiren for type 2 diabetes. $N$ Engl J Med. 2012;367:2204-2213.

64. Bomback AS, Kshirsagar AV, Amamoo MA, et al. Change in proteinuria after adding aldosterone blockers to ACE inhibitors or angiotensin receptor blockers in CKD: a systematic review. Am J Kidney Dis. 2008;51:199-211.

65. Bolignano D, Palmer SC, Navaneethan SD, et al. Aldosterone antagonists for preventing the progression of chronic kidney disease. Cochrane Database Syst Rev. 2014;4:CD007004.

66. Currie G, Taylor AH, Fujita T, et al. Effect of mineralocorticoid receptor antagonists on proteinuria and progression of chronic kidney disease: a systematic review and meta-analysis. BMC Nephrol. 2016;17:127.

67. Bakris GL, Agarwal R, Chan JC, et al. Effect of finerenone on albuminuria in patients with diabetic nephropathy: a randomized clinical trial. JAMA. 2015;314:884-894.

68. Juurlink DN, Mamdani MM, Lee DS, et al. Rates of hyperkalemia after publication of the randomized aldactone evaluation study. N Engl J Med. 2004;351:543-551.

69. Dojki FK, Bakris G. Nonsteroidal mineralocorticoid antagonists in diabetic kidney disease. Curr Opin Nephrol Hypertens. 2017;26:368-374.

70. Jafar TH, Stark PC, Schmid CH, et al. Progression of chronic kidney disease: the role of blood pressure control, proteinuria, and angiotensin-converting enzyme inhibition: a patient-level meta-analysis. Ann Intern Med. 2003;139:244-252.

71. Bakris GL. Slowing nephropathy progression: focus on proteinuria reduction. Clin J Am Soc Nephrol. 2008;3(Suppl 1):S3-10.

72. Palmer BF. Supratherapeutic doses of angiotensin receptor blockers to decrease proteinuria in patients with chronic kidney disease. Am J Nephrol. 2008;28:381-390.

73. O'Hare AM, Hotchkiss JR, Kurella Tamura M, et al. Interpreting treatment effects from clinical trials in the context of real-world risk information: end-stage renal disease prevention in older adults. JAMA Intern Med. 2014;174:391-397.

74. Palmer BF. Managing hyperkalemia caused by inhibitors of the renin-angiotensin-aldosterone system. N Engl J Med. 2004;351:585-592.

75. Rastogi A, Arman F, Alipourfetrati S. New agents in treatment of hyperkalemia: an opportunity to optimize use of RAAS inhibitors for blood pressure control and organ protection in patients with chronic kidney disease. Curr Hypertens Rep. 2016;18:55.

76. Appel GB, Radhakrishnan J, Avram MM, et al. Analysis of metabolic parameters as predictors of risk in the RENAAL study. Diabetes Care. 2003;26:1402-1407.

77. Weir MR, Bakris GL, Bushinsky DA, et al. Patiromer in patients with kidney disease and hyperkalemia receiving RAAS inhibitors. $\mathrm{N}$ Engl J Med. 2015;372:211-221.

78. Goncalves AR, Khwaja A, Ahmed AK, et al. Stopping renin-angiotensin system inhibitors in chronic kidney disease: predictors of response. Nephron Clin Pract. 2011;119:c348-54.

79. Roussel R, Lorraine J, Rodriguez A, et al. Overview of data concerning the safe use of antihyperglycemic medications in type 2 diabetes mellitus and chronic kidney disease. Adv Ther. 2015;32:1029-1064.

80. Sanchez-Rangel E, Inzucchi SE. Metformin: clinical use in type 2 diabetes. Diabetologia. 2017;60:1586-1593.

81. Gudmundsdottir $H$, Aksnes $H$, Heldal $K$, et al. Metformin and antihypertensive therapy with drugs blocking the renin angiotensin system, a cause of concern? Clin Nephrol. 2006;66:380-385.

82. Coca SG, Ismail-Beigi $\mathrm{F}$, Haq N, et al. Role of intensive glucose control in development of renal end points in type 2 diabetes mellitus: systematic review and meta-analysis intensive glucose control in type 2 diabetes. Arch Intern Med. 2012;172:761-769.

83. Scheen AJ. Effects of glucose-lowering agents on renal surrogate endpoints and hard clinical outcomes in patients with type 2 diabetes. Diabetes Metab 2018. In press.

84. Tong L, Adler S. Glycemic control of type 2 diabetes mellitus across stages of renal impairment: information for primary care providers. Postgrad Med. 2018;130:381-393.

85. Scheen AJ. Pharmacodynamics, efficacy and safety of sodium-glucose co-transporter type 2 (SGLT2) inhibitors for the treatment of type 2 diabetes mellitus. Drugs. 2015;75:33-59.

86. Heerspink HJ, Perkins BA, Fitchett DH, et al. Sodium glucose cotransporter 2 inhibitors in the treatment of diabetes: cardiovascular and kidney effects. Potential mechanisms and clinical applications. Circulation. 2016;134:752-772.

- Nice review explaining the potential mechanisms responsible for cardiovascular and renal protection with SGLT2 inhibitors in patients with T2DM.

87. van Bommel EJ, Muskiet MH, Tonneijck L, et al. SGLT2 inhibition in the diabetic kidney-from mechanisms to clinical outcome. Clin J Am Soc Nephrol. 2017;12:700-710.

88. Mima A. Renal protection by sodium-glucose cotransporter 2 inhibitors and its underlying mechanisms in diabetic kidney disease. J Diabetes Complications. 2018;32:720-725.

89. Skrtic M, Cherney DZ. Sodium-glucose cotransporter-2 inhibition and the potential for renal protection in diabetic nephropathy. Curr Opin Nephrol Hypertens. 2015;24:96-103.

90. Mende CW. Diabetes and kidney disease: the role of sodium-glucose cotransporter-2 (SGLT-2) and SGLT-2 inhibitors in modifying disease outcomes. Curr Med Res Opin. 2017;33:541-551.

91. Bonnet F, Scheen AJ. Effects of SGLT2 inhibitors on systemic and tissue low-grade inflammation: potential contribution for diabetic complications and cardiovascular disease. Diabetes Metab. 2018;44:457-464.

92. Scheen AJ. Pharmacokinetics, pharmacodynamics and clinical use of SGLT2 inhibitors in patients with type 2 diabetes mellitus and chronic kidney disease. Clin Pharmacokinet. 2015;54:691-708.

93. Barnett AH, Mithal A, Manassie J, et al. Efficacy and safety of empagliflozin added to existing antidiabetes treatment in patients with type 2 diabetes and chronic kidney disease: a randomised, double-blind, placebo-controlled trial. Lancet Diabetes Endocrinol. 2014;2:369-384.

94. Cherney DZI, Cooper ME, Tikkanen I, et al. Pooled analysis of phase III trials indicate contrasting influences of renal function on blood pressure, body weight, and $\mathrm{HbA} 1 \mathrm{c}$ reductions with empagliflozin. Kidney Int. 2018;93:231-244.

95. Dekkers CCJ, Wheeler DC, Sjostrom CD, et al. Effects of the sodium-glucose co-transporter 2 inhibitor dapagliflozin in patients with type 2 diabetes and Stages 3b-4 chronic kidney disease. Nephrol Dial Transplant. 2018;33:1280.

96. Wanner C, Lachin JM, Inzucchi SE, et al. Empagliflozin and clinical outcomes in patients with type 2 diabetes mellitus, established cardiovascular disease, and chronic kidney disease. Circulation. 2018;137:119-129.

97. Neuen BL, Ohkuma T, Neal B, et al. Cardiovascular and renal outcomes with canagliflozin according to baseline kidney function: data from the CANVAS program. Circulation. 2018;138:1537-1550.

98. Mazidi M, Rezaie P, Gao HK, et al. Effect of sodium-glucose cotransport-2 inhibitors on blood pressure in people with type 2 diabetes mellitus: a systematic review and meta-analysis of 43 randomized control trials with 22528 patients. J Am Heart Assoc. 2017 May 25;6(6):e004007.

99. Scheen AJ, Delanaye P. Effects of reducing blood pressure on renal outcomes in patients with type 2 diabetes: focus on SGLT2 inhibitors and EMPA-REG OUTCOME. Diabetes Metab. 2017;43:99-109.

100. Cherney DZI, Zinman B, Inzucchi SE, et al. Effects of empagliflozin on the urinary albumin-to-creatinine ratio in patients with type 2 diabetes and established cardiovascular disease: an exploratory analysis from the EMPA-REG OUTCOME randomised, placebo-controlled trial. Lancet Diabetes Endocrinol. 2017;5:610-621.

101. Mahaffey KW, Neal B, Perkovic V, et al. Canagliflozin for primary and secondary prevention of cardiovascular events: results from the 
CANVAS program (canagliflozin cardiovascular assessment study). Circulation. 2018;137:323-334.

102. Raz I, Mosenzon O, Bonaca MP, et al. DECLARE-TIMI 58: participants' baseline characteristics. Diabetes Obes Metab. 2018;20:1102-1110.

103. Wiviott SD, Raz I, Bonaca MP, et al. Dapagliflozin and cardiovascular outcomes in type 2 diabetes. N Engl J Med. 2018. published on line; doi:Nov 10, 2018. DOI:10.1056/NEJMoa1812389

. In patients with type 2 diabetes who had or were at risk for atherosclerotic cardiovascular disease, treatment with dapagliflozin did result in a lower rate of hospitalization for heart failure and renal events.

104. Zelniker TA, Wiviott SD, Raz I, et al. SGLT2 inhibitors for primary and secondary prevention of cardiovascular and renal outcomes in type 2 diabetes: a systematic review and meta-analysis of cardiovascular outcome trials. Lancet. 2018 Nov 9; S0140-6736(18)32590-X DOI: 10.1016/S0140-6736(18)32590-X [Epub ahead of print]

-. This meta-analysis of three large prospective trials shows that SGLT2 inhibitors have robust benefits on reducing progression of renal disease regardless of existing atherosclerotic cardiovascular disease or a history of heart failure.

105. Jardine MJ, Mahaffey KW, Neal B, et al. The Canagliflozin and Renal Endpoints in Diabetes with Established Nephropathy Clinical Evaluation (CREDENCE) study rationale, design, and baseline characteristics. Am J Nephrol. 2017;46:462-472.

106. Szalat A, Perlman A, Muszkat M, et al. Can SGLT2 inhibitors cause acute renal failure? Plausible role for altered glomerular hemodynamics and medullary hypoxia. Drug Saf. 2018;41:239-252.

107. Scheen AJ. SGLT2 inhibitors: benefit/risk balance. Curr Diab Rep. 2016;16:92.

108. Wanner C. EMPA-REG OUTCOME: the nephrologist's point of view. Am J Cardiol. 2017;120:S59-S67.

109. Perlman A, Heyman SN, Matok I, et al. Acute renal failure with sodium-glucose-cotransporter-2 inhibitors: analysis of the FDA adverse event report system database. Nutr Metab Cardiovasc Dis. 2017;27:1108-1113.

110. Saly DL, Perazella MA. Harnessing basic and clinic tools to evaluate SGLT2 inhibitor nephrotoxicity. Am J Physiol Renal Physiol. 2017;313:F951-F54.

111. Radholm K, Wu JH, Wong MG, et al. Effects of sodium-glucose cotransporter-2 inhibitors on cardiovascular disease, death and safety outcomes in type 2 diabetes - A systematic review. Diabetes Res Clin Pract. 2018;140:118-128.

112. Neal B, Perkovic V, Mahaffey KW, et al. Canagliflozin and cardiovascular and renal events in type 2 diabetes. N Engl J Med. 2017;377:644-657.

-. The CANVAS program showed that the SGLT2 inhibitor canagliflozin is associated with a lower risk of cardiovascular events and renal outcomes in patients with T2DM and high cardiovascular risk.

113. Scheen AJ. Does lower-limb amputation concern all SGLT-2 inhibitors? Nat Rev Endocrinol. 2018;14:326-328.

114. Franz $D$, Zheng $Y$, Leeper $N J$, et al. Trends in rates of lower extremity amputation among patients with end-stage renal disease who receive dialysis. JAMA Int Med. 2018;178:1025-1032.

115. Muskiet MHA, Tonneijck L, Smits MM, et al. GLP-1 and the kidney: from physiology to pharmacology and outcomes in diabetes. Nat Rev Nephrol. 2017;13:605-628.

116. Marso SP, Bain SC, Consoli A, et al. Semaglutide and cardiovascular outcomes in patients with type 2 diabetes. $\mathrm{N}$ Engl J Med. 2016;375:1834-1844.

117. Holman RR, Bethel MA, Mentz RJ, et al. Effects of once-weekly exenatide on cardiovascular outcomes in type 2 diabetes. $N$ Engl J Med. 2017;377:1228-1239.

118. Pfeffer MA, Claggett B, Diaz R, et al. Lixisenatide in patients with type 2 diabetes and acute coronary syndrome. $\mathrm{N}$ Engl J Med. 2015;373:2247-2257.

119. Hernandez AF, Green JB, Janmohamed S, et al. Albiglutide and cardiovascular outcomes in patients with type 2 diabetes and cardiovascular disease (harmony outcomes): a double-blind, randomised placebo-controlled trial. Lancet. 2018. published on line. DOI:10.1016/S0140-6736(18)32261-X
120. Tuttle KR, Dwight McKinney T, Davidson JA, et al. Effects of once weekly dulaglutide on kidney function in patients with type 2 diabetes in phase II and III clinical trials. Diabetes Obes Metab. 2017;19:436-441.

121. Tuttle KR, Lakshmanan MC, Rayner B, et al. Dulaglutide versus insulin glargine in patients with type 2 diabetes and moderate-to-severe chronic kidney disease (AWARD-7): a multicentre, open-label, randomised trial. Lancet Diabetes Endocrinol. 2018;6:605-617.

122. Cherney DZI, Verma S, Parker JD. Dulaglutide and renal protection in type 2 diabetes. Lancet Diabetes Endocrinol. 2018;6:588-590.

123. Dicembrini I, Nreu B, Scatena A, et al. Microvascular effects of glucagon-like peptide-1 receptor agonists in type 2 diabetes: a meta-analysis of randomized controlled trials. Acta Diabetol. 2017;54:933-941.

124. Gargiulo P, Savarese G, D'Amore C, et al. Efficacy and safety of glucagon-like peptide-1 agonists on macrovascular and microvascular events in type 2 diabetes mellitus: A meta-analysis. Nutr Metab Cardiovasc Dis. 2017;27:1081-1088.

125. Avgerinos I, Karagiannis T, Malandris K, et al. Glucagon-like peptide 1 receptor agonists and microvascular outcomes in type 2 diabetes: a systematic review and meta-analysis. Diabetes Obes Metab. 2018. published on line. DOI:10.1111/dom.13484

126. Perez-Gomez MV, Sanchez-Nino MD, Sanz AB, et al. Horizon 2020 in diabetic kidney disease: the clinical trial pipeline for add-on therapies on top of renin angiotensin system blockade. J Clin Med. 2015;4:1325-1347.

127. Fernandez-Fernandez B, Ortiz A, Gomez-Guerrero C, et al. Therapeutic approaches to diabetic nephropathy-beyond the RAS. Nat Rev Nephrol. 2014;10:325-346.

128. Breyer MD, Kretzler M. Novel avenues for drug discovery in diabetic kidney disease. Expert Opin Drug Discov. 2018;13:65-74.

129. Lacava V, Pellicano V, Ferrajolo C, et al. Novel avenues for treating diabetic nephropathy: new investigational drugs. Expert Opin Investig Drugs. 2017;26:445-462.

130. Cherney DZI, Bakris GL. Novel therapies for diabetic kidney disease. Kidney Int Suppl. 2018;8:18-25.

- Recent review article on new therapies for renal protection in type 2 diabetic patients.

131. de Zeeuw D, Akizawa T, Audhya P, et al. Bardoxolone methyl in type 2 diabetes and stage 4 chronic kidney disease. N Engl J Med. 2013;369:2492-2503.

132. Heerspink HJL, Andress DL, Bakris G, et al. Baseline characteristics and enrichment results from the SONAR trial. Diabetes Obes Metab. 2018;20:1829-1835.

133. Becker GJ, Hewitson TD. Animal models of chronic kidney disease: useful but not perfect. Nephrol Dial Transplant. 2013;28:2432-2438.

134. Samuels J. Use of surrogate outcomes in nephrology research. Adv Chronic Kidney Dis. 2016;23:363-366.

135. Norris KC, Smoyer KE, Rolland C, et al. Albuminuria, serum creatinine, and estimated glomerular filtration rate as predictors of cardio-renal outcomes in patients with type 2 diabetes mellitus and kidney disease: a systematic literature review. BMC Nephrol. 2018;19:36.

136. National Kidney Foundation. KDOQI clinical practice guideline for diabetes and CKD: 2012 update. Am J Kidney Dis. 2012;60:850-886.

137. Nistor I, Bolignano D, Haller MC, et al. Why creating standardized core outcome sets for chronic kidney disease will improve clinical practice. Nephrol Dial Transplant. 2017;32:1268-1273.

138. Coresh J, Turin TC, Matsushita K, et al. Decline in estimated glomerular filtration rate and subsequent risk of end-stage renal disease and mortality. JAMA. 2014;311:2518-2531.

139. Levey AS, Inker LA, Matsushita K, et al. GFR decline as an end point for clinical trials in CKD: a scientific workshop sponsored by the national kidney foundation and the us food and drug administration. Am J Kidney Dis. 2014;64:821-835.

140. Yamanouchi M, Skupien J, Niewczas MA, et al. Improved clinical trial enrollment criterion to identify patients with diabetes at risk of end-stage renal disease. Kidney Int. 2017;92:258-266.

141. Zou H, Zhou B, Xu G. SGLT2 inhibitors: a novel choice for the combination therapy in diabetic kidney disease. Cardiovasc Diabetol. 2017;16:65. 
142. Kalra S, Singh V, Nagrale D. Sodium-glucose cotransporter-2 inhibition and the glomerulus: A review. Adv Ther. 2016;33:1502-1518.

143. Shin SJ, Chung S, Kim SJ, et al. Effect of sodium-glucose co-transporter 2 inhibitor, dapagliflozin, on renal renin-angiotensin system in an animal model of type 2 diabetes. PLoS One. 2016;11:e0165703.

144. Heerspink HJ, Johnsson E, Gause-Nilsson I, et al. Dapagliflozin reduces albuminuria in patients with diabetes and hypertension receiving renin-angiotensin blockers. Diabetes Obes Metab. 2016;18:590-597.

145. Petrykiv S, Laverman GD, de Zeeuw D, et al. Does SGLT2 inhibition with dapagliflozin overcome individual therapy resistance to RAAS inhibition?. Diabetes Obes Metab. 2018;20:224-227.

146. Zinman B, Wanner C, Lachin JM, et al. Empagliflozin, cardiovascular outcomes, and mortality in type 2 diabetes. $\mathrm{N}$ Engl J Med. 2015;373:2117-2128.
147. Tang H, Li D, Zhang J, et al. Sodium-glucose co-transporter-2 inhibitors and risk of adverse renal outcomes among patients with type 2 diabetes: A network and cumulative meta-analysis of randomized controlled trials. Diabetes Obes Metab. 2017;19:1106-1115.

148. Scheen AJ. Cardiovascular outcome studies in type 2 diabetes: comparison between SGLT2 inhibitors and GLP-1 receptor agonists. Diabetes Res Clin Pract. 2018;143:88-100.

149. Davies MJ, D'Alessio DA, Fradkin J, et al. Management of hyperglycaemia in type 2 diabetes, 2018. A consensus report by the American Diabetes Association (ADA) and the European Association for the Study of Diabetes (EASD). Diabetologia. 2018;61:2461-2498.

150. Vupputuri S, Kimes TM, Calloway MO, et al. The economic burden of progressive chronic kidney disease among patients with type 2 diabetes. J Diabetes Complications. 2014;28:10-16. 